\title{
Elementary heating events - magnetic interactions between two flux sources
}

\section{Rates of flux reconnection}

\author{
C. E. Parnell ${ }^{1}$ and K. Galsgaard ${ }^{1,2}$ \\ 1 School of Mathematics and Statistics, University of St Andrews, St Andrews, KY16 9SS, Scotland \\ e-mail: clare@mcs.st-and.ac.uk \\ ${ }^{2}$ Niels Bohr Institute for Astronomy, Physics and Geophysics, Julie Maries vej 31, 2300 Copenhagen Ø, Denmark
}

Received 18 September 2003 / Accepted 20 August 2004

\begin{abstract}
Magnetic fragments in the photosphere are in continuous motion and, due to the complex nature of the magnetic field in the solar atmosphere, these motions are likely to drive a lucrative coronal energy source: the passing of initiallyunconnected opposite-polarity fragments that release energy through both closing and then re-opening the same fieldlines. Three-dimensional, time-dependent MHD and potential models are used to investigate the passing of fragments in an overlying field.

The processes of closing and opening the field generally occur through separator and separatrix reconnection, respectively. The rates of flux reconnection in these processes are determined. They are found to be dependent on the direction of the surrounding magnetic field relative to the motion of the fragments and the velocity of the sources. In particular, separator reconnection rates (closing) and separatrix-surface reconnection rates (opening) are directly related to the rate of flux transport perpendicular to the current sheet (overlying field). The results suggest that both types of reconnection are fast with the peak rates of separator and separatrix reconnection occurring at $58 \%$ and $29 \%$ of the peak potential reconnection rate, respectively, when the sources are driven at a hundredth of the peak Alfvén velocity in the box. Moreover, the slower the system is driven the closer the flux reconnection rates are to the instantaneous potential rates. Furthermore, there is a maximum reconnection rate for both types of reconnection as the driving speed tends to the Alfvén speed with the separatrix reconnection rate typically half that of separator reconnection. These results suggest that, on the Sun, reconnection driven by the passing of small-scale network and intranetwork fragments is a highly efficient process that is very likely to contribute significantly to the heating of the background solar corona.

The three-dimensional reconnection processes are efficient because, unlike in two-dimensions, there are many places within the current sheets where reconnection can take place simultaneously giving rise to fine-scale structure along the boundaries between the open, closed and re-opened flux. Furthermore, due to the complexity of the magnetic field above the photosphere the reconnection all takes place low down at less than a quarter of the separation of the initial fragments above the photosphere.
\end{abstract}

Key words. Sun: photosphere - Sun: magnetic fields - magnetohydrodynamics (MHD)

\section{Introduction}

A key question in plasma physics for any process involving reconnection has always been what is the rate of reconnection? The answer to this question is not only important when the process being modelled is a solar flare, which requires $10^{30} \mathrm{ergs}$ of energy to be released over a period of a few minutes, but is also important in the seemingly less dynamic events of bright points, microflares and nanoflares. Here, magnetic energy must still be released in a fraction of the typical magnetic diffusion time. In this paper, we focus on a model for energy release in bright points, microflares and nanoflares. In particular, we investigate the rate of reconnection and the details of the flux evolution.

Possible heating mechanisms for the solar corona have been studied for several decades and the idea that energy is released through magnetic reconnection driven by the interactions of opposite-polarity magnetic fragments is not a new one. Magnetic reconnection can be driven by several different types of photospheric fragment interaction. For instance, magnetic reconnection driven by the emergence of new flux, on both large and small scales, has been explored by many authors (e.g., Uchida \& Sakurai 1977; Forbes \& Priest 1984; Shibata et al. 1992; Dreher et al. 1997; Yokoyama \& Shibata 2001; Zhang \& Low 2002). The reverse process, that is the driving of magnetic reconnection through cancellation (the convergence and mutual loss of flux from opposite polarity magnetic fragments), has also been considered (e.g., Priest et al. 1994; Parnell et al. 1994; Birk et al. 1996). However, there is a third, possibly more significant, mechanism of driven magnetic reconnection that has not been properly investigated. In this mechanism, flux is neither created nor destroyed. 
During the interaction of initially unconnected fragments of opposite polarity that simply pass each other energy is released due to the creation of fieldlines that connect the fragments and then, as the fragments continue moving past each other, through the breaking of these connected fieldlines that have just been formed. Although this is essentially Faraday's idea of "magnetic attraction", which is the basis of magnetodynamics, there has been little analysis done, and few numerical experiments run, to understand exactly how and at what rate energy is released from this process. The process has similarities to the "braiding of fieldlines" suggested by Parker (1987), which has been found to create current sheets in even simpler initial fields than are used in this paper (Galsgaard \& Nordlund 1996; Hendrix \& Van Hoven 1996; Longbottom et al. 1998; Ng $\&$ Bhattachrjee 1998). Clearly, such interactions are the building blocks of more complex magnetic geometries.

Galsgaard et al. (2000) (hereafter, known as Paper I) modelled the interaction of two opposite polarity magnetic fragments passing in an overlying field. This paper focussed on the global topological evolution of the system and identified that two distinct types of reconnection occur in such interactions. Here, however, we look further than answering questions relating to simply coronal heating or even solar physics, but seek to answer a key question of plasma physics, namely, "How does the rate of flux reconnection in three dimensions scale"? There are, of course, many factors that effect the reconnection rate of a driven system: the rate of driving; the direction and strengths of the magnetic field involved; the resistivity; and the boundary conditions of the system. In this paper, we run two sets of experiments to determine the dependence of the rate of flux reconnection on the speed of driving and the relative direction of the magnetic field with respect to the direction of driving. First, however, for completeness we introduce some of the terminology used in this paper and briefly explain the two types of reconnection that arise.

The flux that extends from a magnetic source is usually confined due to surrounding magnetic fields. The volume this flux covers is called, here, the source's flux lobe. It is separated from flux from the other like-polarity sources by a separatrix surface (e.g. Greene 1988; Lau \& Finn 1990; Priest \& Titov 1996) if there is a magnetic null point in the plane of the sources. If there are no nulls a similar structure exists, known as a quasi-separatrix layer (Priest \& Démoulin 1995; Titov et al. 2002; Galsgaard et al. 2003). In this paper, null points are present in the plane of the sources so genuine separatrix surfaces exist. The area covered by a source's separatrix surface is the area of magnetic attraction around that source. A separatrix surface contains fieldlines that extend from a source to a null point (or vice versa). If separatrix surfaces from two opposite-polarity sources overlap then the intersection of the two separatrix surfaces leads to the creation of a separator, a special fieldline linking two null points. Such a situation involves at least four topologically distinct regions enclosing (i) closed flux connecting the two sources; (ii) two open flux lobe's: one extending from each source and (iii) the surrounding overlying magnetic field. If the sources are moved a current sheet may develop along the separator resulting in separator reconnection (e.g.,
Lau \& Finn 1990; Priest \& Titov 1996; Galsgaard \& Nordlund 1997; Galsgaard et al. 2000b). However, reconnection can also occur where there is simply a separatrix surface. This reconnection, known as separatrix reconnection, occurs in current sheets that form on the separatrix surface. There have been many discussions in the magnetospheric literature as to whether reconnection in such current sheets occurs (i) at null points that form in the sheet or (ii) component wise at points where only certain components of the magnetic field cancel (e.g., Shi et al. 1991; Otto 1995, and references therein). Clearly, the specific shape of a separatrix surface or separator, and hence any associated current sheets, will depend mainly on the relative strengths and polarities of the surrounding magnetic sources, but if the evolution of the magnetic field is magnetohydrodynamic then it will also depend on the flows, resistivity and viscosity in the system.

Longcope (1998) used the minimum current corona approach to investigate the interaction of two point sources of opposite polarity embedded in an overlying field. Here, free energy is allowed to accumulate in a single current sheet along the separator while the remaining domain is potential. Depending on the direction and strength of the overlying field and the ratio of strengths of the two point sources the areas over which they interact magnetically can be calculated. Clearly, no matter how close the sources are, if the overlying field is strong enough and in the right direction, then the two sources will not be connected. In general, however, most other angles of the overlying field result in a connection of the sources over a range of separations depending on the strength of the overlying field. A simple test using potential fields gives us some idea as to when sources will connect and disconnect as they pass by each other. However, on the Sun itself, reconnection is not perfect and instantaneous and magnetic sources do not, in general, connect exactly when, in a potential case, they would. Observational evidence supporting this has been recently found by Nandy et al. (2003), and reveals an exponential decay time for active regions to lose energy and relax towards linear forcefree magnetic configurations.

As already mentioned, in Paper I a numerical experiment considering one situation where two magnetic sources pass each other has been carried out. The situation investigated is one in which two opposite-polarity magnetic sources, which are initially unconnected, are driven past each other along an axis that is at right angles to a uniform overlying field. See Fig. 3 for snapshots of the fieldline and current sheet evolution. The details of how the two sources actually interact are investigated. Firstly, the two sources are driven causing the two flux lobes from these sources to be pushed into the overlying field which moves up and over these lobes. As the sources are driven further past each other more and more of the overlying field ahead of them is lifted up out of the way until their flux lobes come head-to-head. Now the tails of these flux lobes themselves are lifted up by the opposite source being driven underneath causing the two lobes to become entwined about each other. This results in the field from the two sources being inclined along oppositely directed slopes leading to current formation down the entire leading sides of the separatrix surfaces surrounding the flux lobes. The current sheet created 
is twisted and lies along the separator between the two lobes. In regions of enhanced current, and therefore enhanced resistive effects, separator reconnection starts and the two sources begin to connect. As the driving continues the current sheet becomes more twisted, more reconnection occurs, and more flux connects the two sources. This closing process continues even when the two sources have moved well beyond their theoretical potential magnetic-disconnection distance. Eventually, the process of re-opening the field again begins, but it is slow compared to the rate of closing and its onset later than the time of re-opening in the potential case. Re-opening occurs through separatrix reconnection at weak current sheets which form on the leading sides of the separatrix surfaces resulting in the slow disconnection of the sources. Note, by a separatrix surface we are referring to any surface that divides topologically distinct regions and can include both traditional fan surfaces with a null (Priest \& Titov 1996) or quasi-separatrix layers (Priest \& Démoulin 1995).

Paper I focussed on determining the mechanisms through which the two sources are connected and unconnected demonstrating that first separator reconnection and then separatrixsurface reconnection, and hence the release of magnetic energy, can occur through such a scenario. Clearly, though a key question is what is the rate of reconnection in both of these mechanisms? In this paper, we determine the individual rates of these reconnection processes in the above experiment and in a series of new experiments. The new experiments investigate the effects of varying the angle of the overlying field and the rate of driving the sources such that the scaling of the reconnection rates can be determined. The effects of the reconnection processes on the internal flux of the sources is also investigated. To put the dynamical MHD results into context we compare them with equivalent potential models in which the potential field from the same sources is derived inside a closed box and evolves over time assuming instantaneous perfect reconnection occurs. This enables us to determine exactly what characteristics are associated with the MHD nature of the experiments rather than being due to simple electromagnetic evolution.

The potential and dynamic MHD models used are described in Sect. 2. The evolution of the global flux of these systems are discussed in Sect. 3. Also in this section we determine the ranges of overlying field angles that permit the creation of connected flux between the two opposite-polarity magnetic sources. The rates of creating this closed flux and re-opening it again are calculated for the different overlying field angles and for different driving speeds. Furthermore, the internal source flux is analysed and the flux regions within the sources which close and re-open are identified and tracked to see how they evolve. Finally, in Sect. 4, we discuss the implications of our results.

\section{Potential and dynamical models}

The particular scenarios investigated are the following. A threedimensional box, non-dimensionalised such that the base is of size $1 \times 1$ and height 0.25 , is studied using a cubed grid of data points of size $128 \times 128 \times 65$, respectively, for most of the experiments, whilst runs with a resolution of $256 \times 256 \times$ 129 have been computed for selected experiments to check the dependence on numerical resolution. On the base $(z=0)$ of the box the normal components of the magnetic field of the positive and negative sources are prescribed. These sources are centred initially at $(0.33,0.33)$ and $(0.66,0.66)$, respectively, have radii of $r_{0}=0.065$ and have peak normal components of magnetic field of $B_{0}=0.85$. The normal component of the source's field has a cosine profile such that the internal flux within the sources peaks at the centre of the source and falls off as $B_{z}=B_{0}\left(1+\cos \left(\pi r / r_{0}\right)\right) / 2, r<r_{0}$, where $r$ is the distance from the source centre.

An overlying horizontal, uniform field is added to this field inclined at an angle $\theta$ to the $y$-axis. For one set of experiments each scenario investigated has a different angle of $\theta$ with the range of angles investigated lying between $-\pi / 2$ and $\pi / 2$ at intervals of $\pi / 10$. In the second set of experiments $\theta$ is held fixed at zero. Note, Paper I only considered the situation where $\theta=0$. The overlying horizontal field strength is held fixed at 0.12 times the strength of the initial peak normal field in the sources in all the experiments.

The sources are driven past each other parallel to the $x$-axis. In the first set of scenarios this speed is fixed at 0.02 times the peak Alfvén speed measured at the base in the sources. In the second set, which have the fixed overlying field angle of $\theta=0$, the driving speed is varied and equals $0.01,0.015,0.02,0.03$ and 0.04 times the peak Alfvén velocity in the box.

\subsection{Potential model}

For the potential model, the boundaries on the unit box are closed in the $x$ and $y$ directions and on the top (in the $z$ direction), i.e. there is no flux across these boundaries. The base contains two sources as described above. Since the magnetic field, $\boldsymbol{B}$, is potential it must always satisfy the equation

$\nabla \times \boldsymbol{B}=\mathbf{0}$.

Hence, $\boldsymbol{B}$ is rewritten in terms of a scalar potential $\phi, \boldsymbol{B}=\nabla \phi$, to ensure that this condition is always met. Furthermore, as the solenoidal condition, $\nabla \cdot \boldsymbol{B}=0$, has to be fulfilled, this requires that $\phi$ must satisfy

$\nabla^{2} \phi=0$

Thus, the three-dimensional magnetic field in the box is found by solving Eq. (2) with the prescribed boundary conditions for the scalar potential, $\phi$, using a linear multigrid method with a Gauss-Seidel smoother. This gives a dipole field in the closed box, which is altered by adding the constant overlying field mentioned above.

For the potential model, the sources are moved across the box with the potential magnetic field recalculated for every new position. Clearly, as the sources get close to the boundaries of the box, edge effects come into play and these affect the resulting magnetic field, as seen in the results mentioned later. 


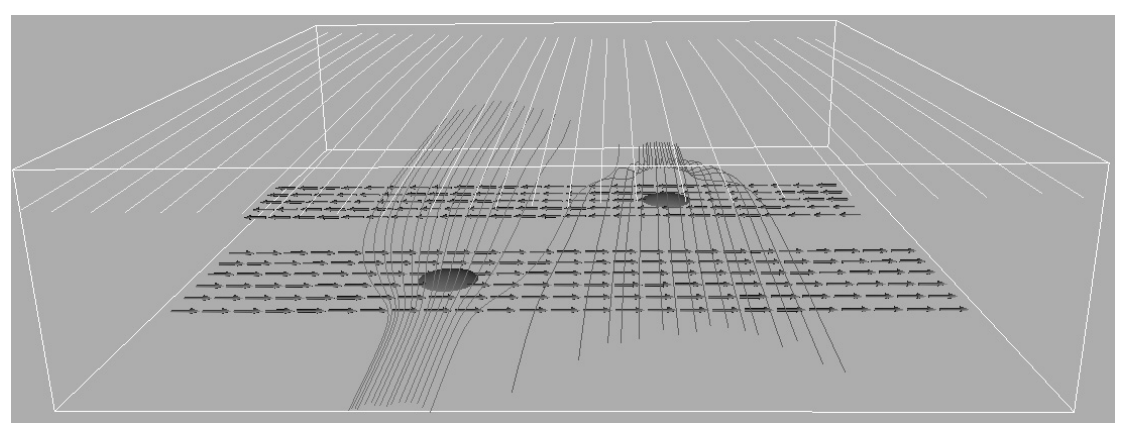

Fig. 1. The initial configuration of the magnetic field and the structure of the imposed driving field. The magnetic field lines represent the boundaries between the different flux lobes and the overlying magnetic field. The vectors on the bottom boundary indicate the direction and structure of the driving field.

\subsection{Dynamical MHD model}

The dynamical MHD code used to consider the interaction of the opposite-polarity magnetic sources, is basically the same as that used in Paper I. The only difference being that previously $\nabla \cdot \boldsymbol{B}=0$ was not properly conserved at the boundaries, but here the algorithm has been changed such that it is. This improvement, although important, has little effect on the dynamic evolution of the magnetic field. To make this paper selfcontained, we give both a brief description of the model and the equations used.

The boundaries on the box are periodic in the $x$ and $y$ directions and closed in the $z$ direction. On the base $(z=0)$ of the box the normal component of the magnetic field is prescribed, as discussed above.

An initial magnetic field is derived by determining the potential magnetic field due to the sources at the base assuming that no flux penetrates the other walls of the box, using the code described above. The overlying field is then added to (partially) break the connections between the sources. This is, however, in contradiction to the periodic boundaries assumed on the box used for the dynamical evolution. This generates weak currents at these walls, creating small-amplitude waves which propagate through the box. This change in the vertical boundary conditions does not significantly influence the magnetic field structures that we are interested in which occur near the centre of the box. The change in the boundary conditions between the potential and dynamical model works because the magnetic field strength falls off very fast with increasing distance from the sources (on the order of $1 / r^{2}$ ). Therefore, within in a very short distance from the sources the added constant magnetic field dominates over the dipole field such that the vertical boundary condition mismatch represents only an insignificant change to a constant magnetic field.

Initial experiments in a unit cubed box revealed that all the reconnection occurred low down within the box because of the rapid fall off in magnetic field strength of the dipole. So to allow a higher numerical resolution in the $z$ direction only the lower quarter of that domain is used in the calculations shown here. In relation to the Sun, if we assume the sources in the experiments have a diameter of $0.5-2 \mathrm{Mm}$, then the nearest distance between them will be $1.5-5 \mathrm{Mm}$ and the typical height of the center of the current sheet will be about $0.5-2 \mathrm{Mm}$ above the photosphere so around the old traditional lower boundary of the solar corona of $1-2 \mathrm{Mm}$.

The driving velocity mentioned above is only applied to specific regions on the base for the dynamic model and is ramped up to a constant velocity. It is applied in just two strips along the base that run parallel to the $x$-axis and are just wider than the diameter of the sources. Outside these regions the velocity is ramped down over a short distance to zero. Since the magnetic field only penetrates the base at the locations of the sources, only they are moved by the imposed driver. Figure 1 shows the initial magnetic field configuration and the structure of the imposed boundary driving.

The equations that govern the dynamic evolution of the magnetic fields are a non-dimensionalised, non-ideal form of the MHD equations and can be written,

$$
\begin{aligned}
& \frac{\partial \rho}{\partial t}=-\nabla \cdot \rho \boldsymbol{u} \\
& \frac{\partial \rho \boldsymbol{u}}{\partial t}=-\nabla \cdot(\rho \boldsymbol{u} \boldsymbol{u}+\underline{\underline{\tau}})-\nabla P+\boldsymbol{j} \times \boldsymbol{B} \\
& \frac{\partial e}{\partial t}=-\nabla \cdot(e \boldsymbol{u})-P \nabla \cdot \boldsymbol{u}-\rho\left(T-T_{0}\right) / t_{\text {cool }}+Q_{\text {joule }}+Q_{\text {visc }} \\
& \frac{\partial \boldsymbol{B}}{\partial t}=-\nabla \times \boldsymbol{E} \\
& \boldsymbol{E}=-(\boldsymbol{u} \times \boldsymbol{B})+\eta \boldsymbol{j} \\
& \boldsymbol{j}=\nabla \times \boldsymbol{B} \\
& P=e(\gamma-1)
\end{aligned}
$$

where the vectors $\boldsymbol{B}, \boldsymbol{u}$ and $\boldsymbol{J}$ are the magnetic field, velocity and current, respectively; $\underline{\tau}$ is the viscous stress tensor; the scalars $\rho, P, T, T_{0}, Q_{\text {joule }}, Q_{\mathrm{visc}}$ and $e$ are the density, plasma pressure, temperature, equilibrium temperature, joule dissipation, viscous dissipation and thermal energy, respectively. The constants $t_{\text {cool }}, \eta$ and $\gamma$ are the exponential cooling time (here, set to 1.64 Alfvén crossing times), magnetic resistivity and the ratio of specific heats which, in an ideal gas, is assumed to be $5 / 3$. To non-dimensionalise the equations the magnetic permeability $\mu_{0}$ is set to 1 and the gas constant, $R$ is set to $\mu$, the mean molecular weight. We assume that the initial plasma is at rest at a constant temperature and has a density equal to $1 / 2$ and the initial plasma pressure is set to $1 / 6$. In this paper, the time units are scaled to the Alfvén crossing time of the box derived 
for the peak magnetic field strength of the sources at the bottom boundary.

To solve the equations a sixth-order method is used to derive the spatial derivatives and a third-order predictor-corrector method for advancement in time. A staggered grid in a Cartesian box is adopted, with a fifth-order method for interpolation between points on the grids, which fulfills the conservation laws of MHD. To achieve the highest spatial resolution for the given numerical resolution viscous and magnetic resistivity are both handled using combined second and fourth-order methods in connection with a discontinuous capture mechanism (Nordlund \& Galsgaard 1997; www@astro.ku.dk/ kg). This implies that the effective magnetic Reynolds numbers become both space and time dependent.

The value of the magnetic resistivity, $\eta$, can be estimated at each gridpoint using the joule dissipation in the code, assuming the traditional expression for this dissipation of $\eta j^{2}$. This has been done at the most dynamically active time for two experiments, which are the same except for their resolution. The derived $\eta$ s are then used to estimate Lundquist numbers $(S)$ for the experiments. It is found that the resulting values of $S$ are of the order of $2 \times 10^{4}$ and $2 \times 10^{5}$ for the $128 \times 128 \times 65$ and $256 \times 256 \times 129$ runs, respectively. Depending on the precise way in which the averaging is done, these numbers can change within a factor of 10. Furthermore, tests on similar codes comparing constant and hyper resistivity suggest that hyper resistivity allows considerably sharper gradients in the magnetic field to form creating larger currents and more well defined current sheets than in constant resistivity codes. Clearly, the values of $S$ obtained are many orders of magnitude below the Lundquist number expected in the solar corona, however, what is important is that they are many orders of magnitude greater than unity. This means that the general behaviour observed in these experiments and the dependences discovered between the parameters in the models and the reconnection rates are highly likely to still be valid in the solar corona. What would, of course, change is the factors that multiply these parameters and this must be kept in mind when reviewing the results.

\section{Flux connectivity and magnetic reconnection}

In the experiments, as the strength and direction of the overlying field changes so does the fraction of closed flux connecting the sources. The initial magnetic topology also varies, having either three or four independent flux regions. The fraction of closed flux, and the rate of closing this flux, in each experiment depends on (i) the relative strengths of the overlying field and the sources; (ii) the relative direction of the overlying field to the line through the centre of the sources; (iii) the relative direction of the overlying field to the direction of transport and (iv) the speed of transport of the sources. In Paper I, the situation studied had the overlying field and direction of transport at right angles to each other and had an initial set up where the line through the source centres bisected this angle. The initial setup in all the experiments investigated here have the line through the source centres inclined at $\pi / 4$ to the direction of the source driver. However, the direction of the overlying field ranges from parallel to anti-parallel to the direction of transport, in the first set of experiments, allowing us to investigate the effect of varying the relative directions of the overlying field to both the line through the source centres and the direction of their transport. In a second set of experiments the direction of the overlying field is held fixed at right angles to the direction of transport, however, the speed of the imposed driving velocity is varied.

\subsection{Magnetic topology}

Snapshots of the magnetic topology from four experiments with varying overlying field angles ranging from $\theta=$ $-\pi / 5,0,3 \pi / 10$ and $\pi / 2$, respectively, are shown in Figs. $2-5$. The dynamical evolution is shown by the frames in the lefthand column and the potential evolution by those on the right. The snapshots are taken every 5 Alfvén times starting with the snapshots in row 1 taken at $t=0$ through to those taken at $t \approx 35$ in row 8 . In all the snapshots fieldlines are traced from the same points inside the sources and from the same points in the overlying field above. Also the maximum and minimum currents, are normalised in each experiment such that the same isosurface is shaded in each frame allowing the time development of the current sheets in the dynamical experiments to be seen. The current isosurface shown has approximately half the overall maximum current magnitude of each experiment. No current sheets are seen in the potential experiments, which evolve through a series of equi-potential states, since any currents are assumed to be immediately dissipated giving rise to instantaneous and perfect reconnection. This means the processes of closing or re-opening the magnetic field in the potential experiments occur much more rapidly and more completely than in the dynamical ones, as seen below.

In Fig. 2, $\theta=-\pi / 5$, the flux lobes from each source are angled away from the other source. This means, in both the potential and dynamical evolution, that as the two sources are driven past each other, it is not until the two sources have moved beyond their minimum separation that their flux lobes first touch. In deed, in the potential experiment, closed field is visible between the two sources after just 10 Alfvén times (right frame 3) with the sources having completely closed and re-opened again in less than 25 Alfvén times from the start of the driving (right frame 6). In contrast, in the dynamically experiment, there is a delay with the flux lobes first become entwined about each other leading to the formation of a twisted current sheet after 20 Alfvén times. This current sheet rapidly grows and extends along the full length of the interface between the two lobes and is clearly twisted (left frame 6) outlining exactly how much the two flux lobes have interlocked with each other. As the two sources are driven further separator reconnection occurs at the current sheet resulting in closed magnetic field connecting the two sources. The advection of the sources and the interlocking of the flux lobes injects magnetic helicity into the system. As magnetic reconnection conserves helicity, the new closed field lines must be twisted. Reconnection at this current sheet continues, after the driving has stopped (at 27 Alfvén times), until the current in the current sheet is dissipated and a new quasi-static state is reached. 

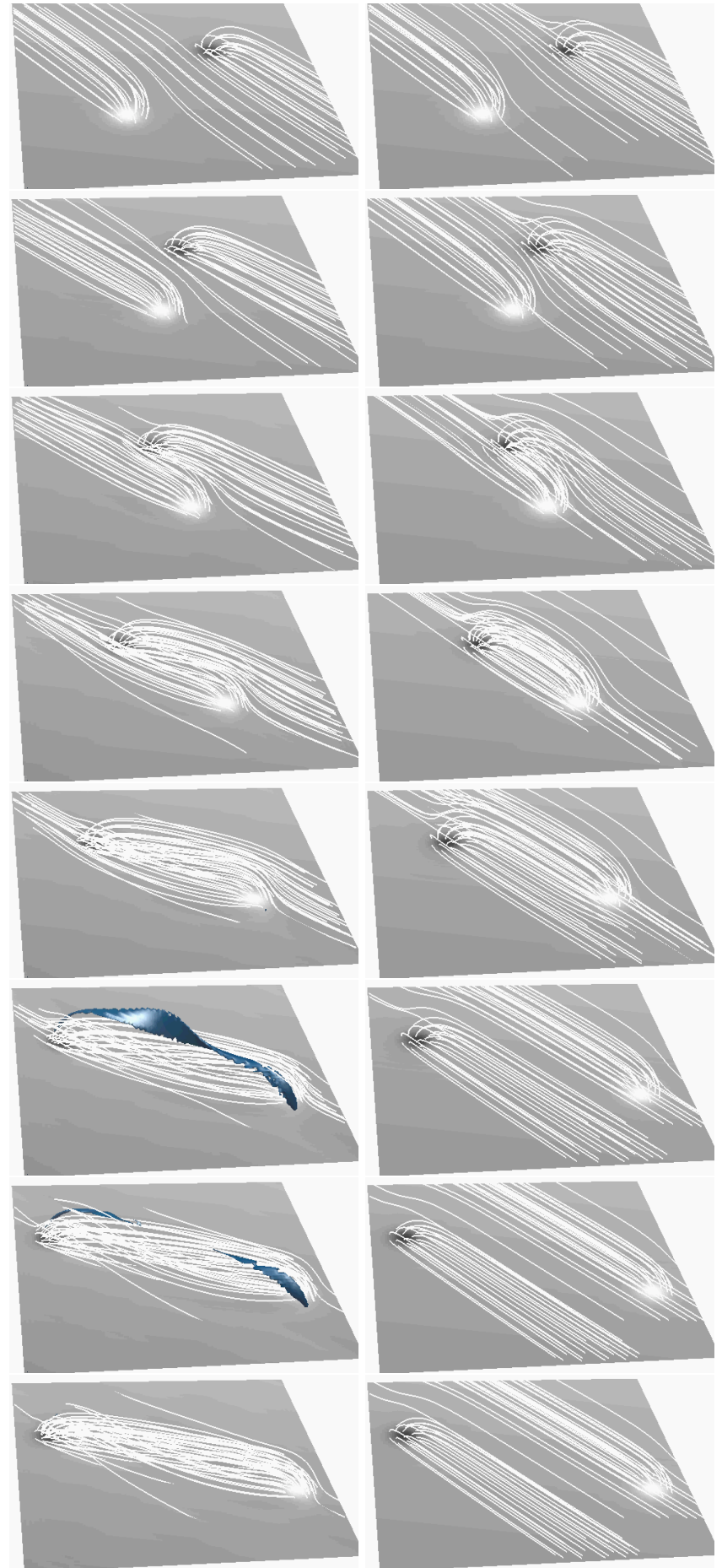

Fig. 2. The time evolution (down each column) of the magnetic field (white lines) and current isosurfaces (dark surfaces) for the dynamical (left) and potential (right) experiments with overlying field angle $\theta=-\pi / 5$ and driving velocity $v_{\mathrm{d}}=0.02$. The times of the frames are from $t=0$ through to $t=35$ Alfvén crossing times, in steps of 5 Alfvén times.

The evolution of the magnetic field in Fig. 3 initially follows a similar pattern, but towards the end the closed flux from the sources also starts to re-open. This is possible since the angle of the overlying field, $\theta=0$, means that the flux lobes, although initially unconnected, come into contact much earlier
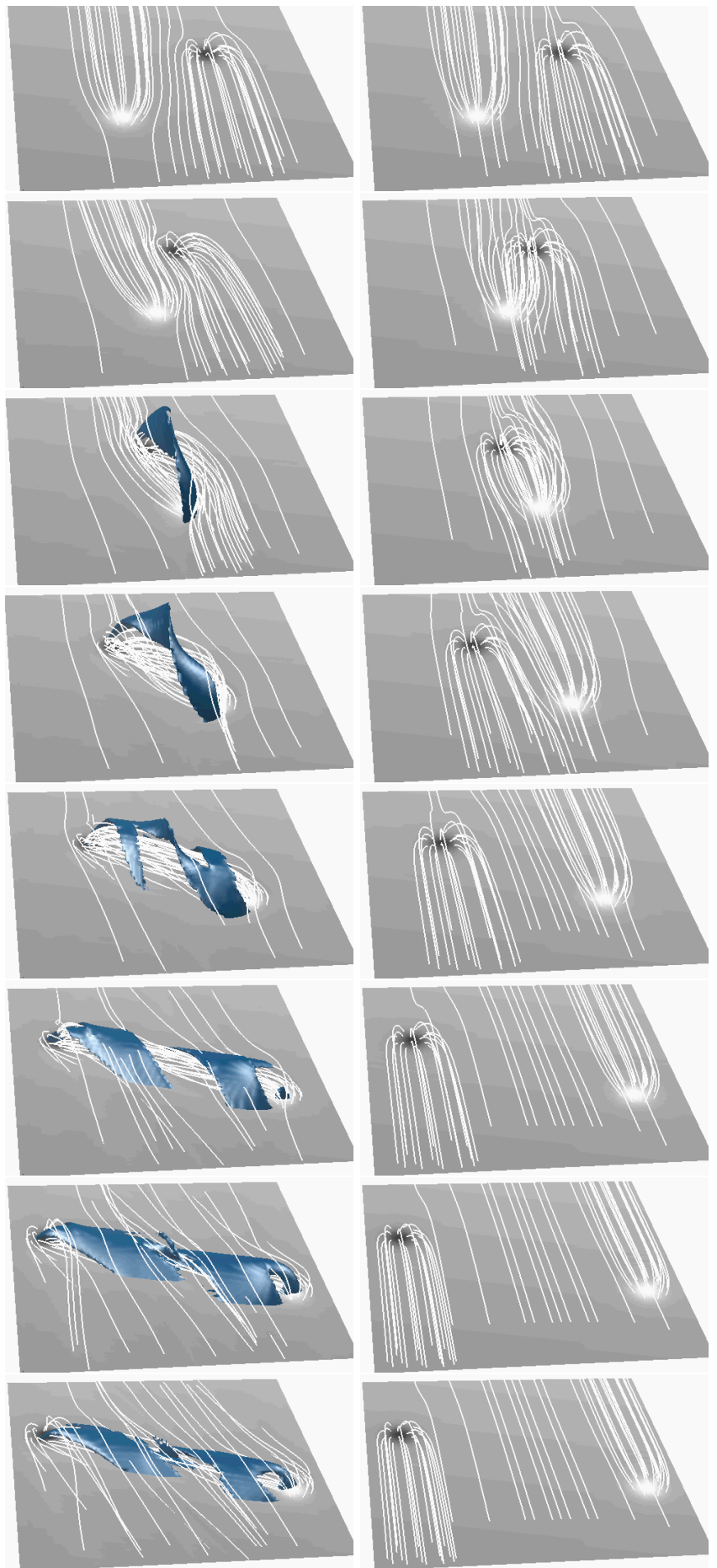

Fig. 3. As Fig. 2 but with overlying field $\theta=0$ and driving velocity $v_{\mathrm{d}}=0.02$.

during the experiment. In fact, in the potential experiment, the sources are well connected before 5 Alfvén times (right frame 2) and before 10 Alfvén times (left frame 3 ) in the dynamical experiment. A twisted current sheet, which is formed in exactly the same way as in the previous experiment, is clearly seen here extending between the two sources. This sheet grows and becomes more twisted as the two sources continue to be driven. Separator reconnection is driven at this current sheet and enables a large amount of closed flux to form between the two sources. 

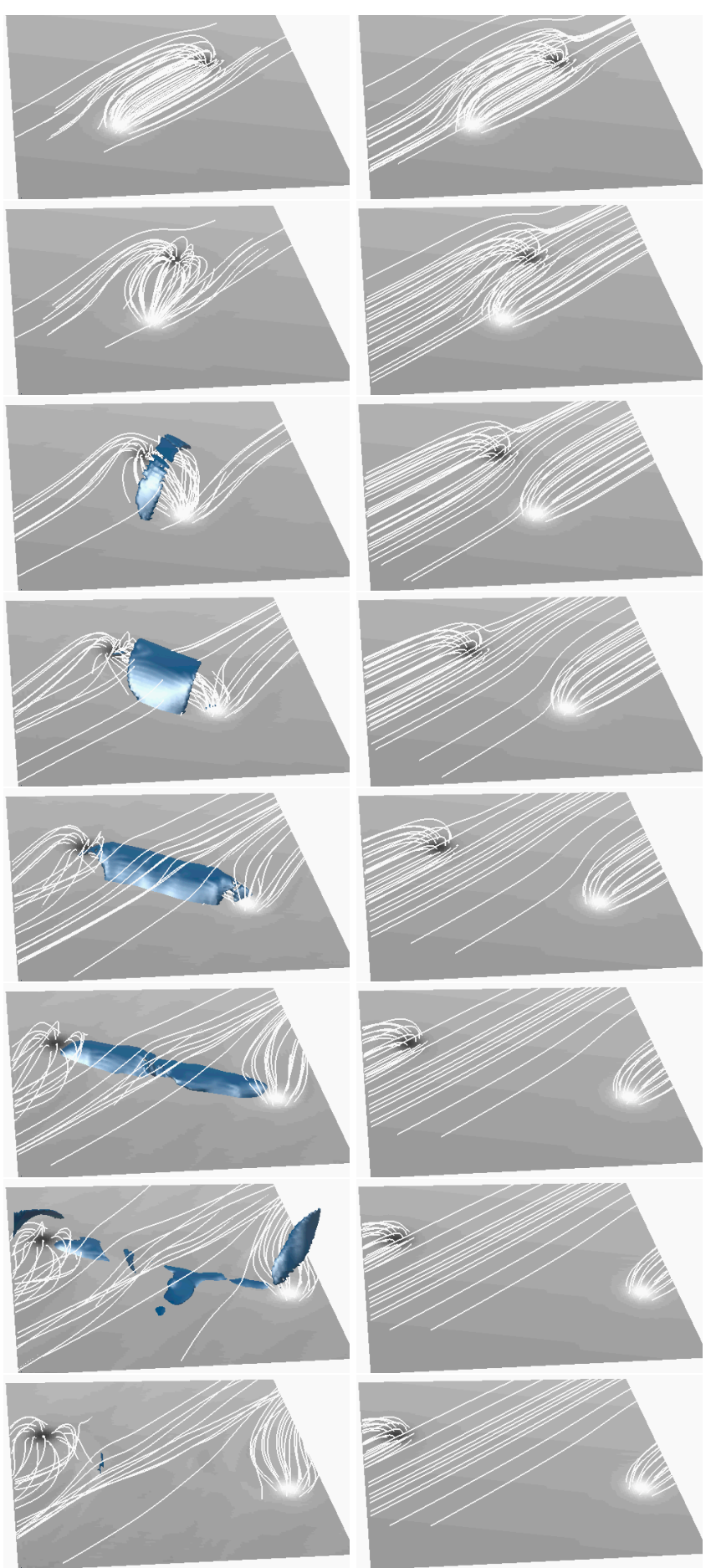

Fig. 4. As Fig. 2 but with overlying field $\theta=3 \pi / 10$ and driving velocity $v_{\mathrm{d}}=0.02$.

In the potential regime neither the closing nor re-opening phases last long. In fact, by 15 Alfvén time units the closed flux from the sources has all but re-opened (right frame 4). From these frames alone it is not possible to say if there is any overlap in the closing and re-opening processes. On the other hand, in the dynamical regime there is a clear overlap of these processes. In frame 5 (left), the twisted current sheet is still visible indicating that separator reconnection is still taking place. However, two other strips of current have now also formed.

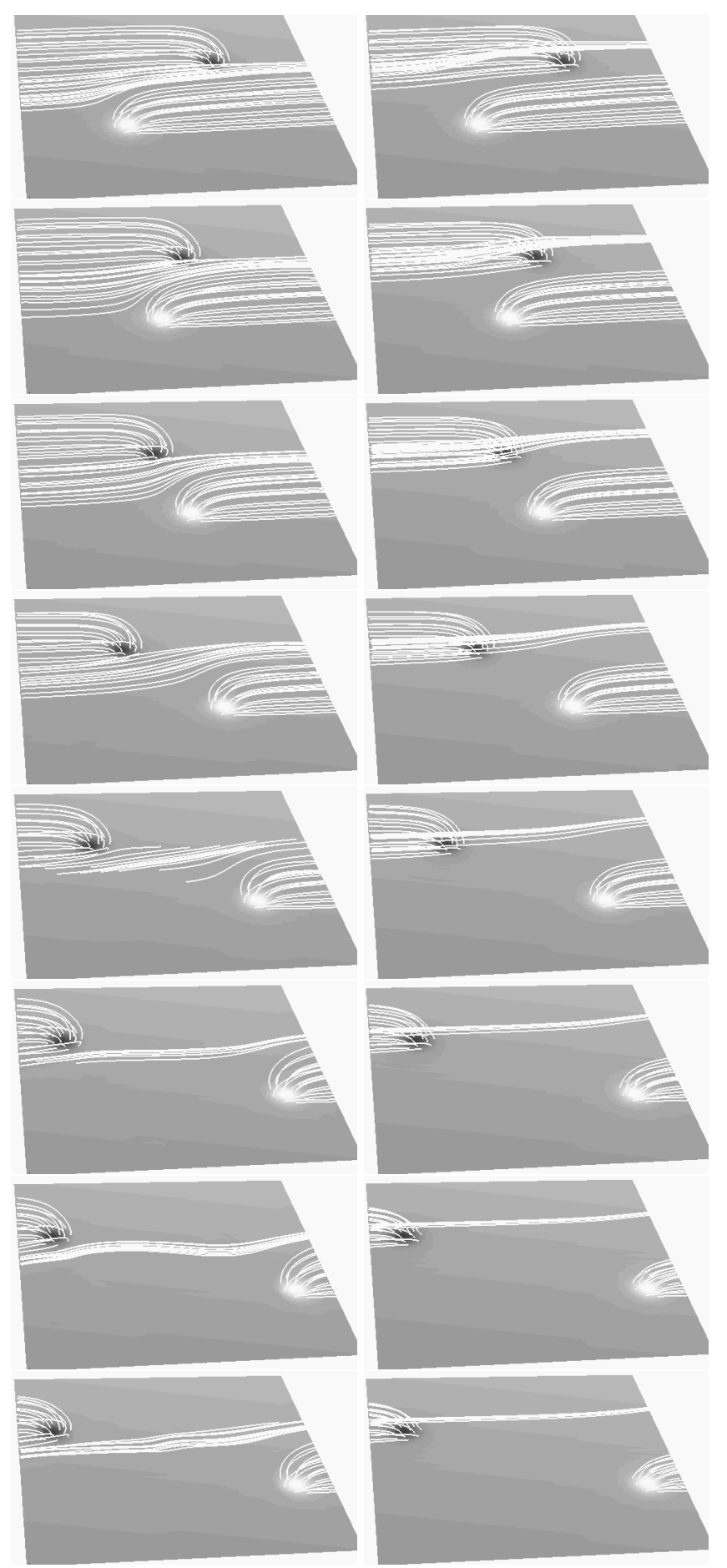

Fig. 5. As Fig. 2 but with overlying field $\theta=\pi / 2$ and driving velocity $v_{\mathrm{d}}=0.02$.

These strips extend across the width of the closed flux lobe lying more-or-less parallel with the overlying field. They mark the start of the re-opening reconnection process. In the next few frames (left frames 6-8) the separator reconnection draws to an end, signaled by the shrinking of the twisted current sheet. Instead the flattish current strips widen, which they continue to do over the remaining frames until they encompass most of the closed flux lobe, enabling the re-opening of the closed field to progress through separatrix-surface reconnection. After the 
driving stops at 27 Alfvén times and the field relaxes towards an equilibrium state with both closed and re-opened magnetic field.

In the third scenario, Fig. 4, the overlying field is inclined at $\theta=3 \pi / 10$ allowing the flux sources to start off partly (70\%) connected resulting in initially 4 topologically distinct domains. Thus, here only the process of re-opening occurs. In the potential evolution the re-opening of this field takes less than 10 Alfvén times (right frames 1-3), whereas in the dynamical experiment it takes 35 Alfvén times (left frames 1-8). In deed, the dynamical re-opening process does not start immediately. Instead, as the sources are driven, the closed flux lobe first rotates such that it lies at an angle to the overlying field before a current sheet forms along the separator line lying parallel to the overlying field and encompassing the closed flux lobe (left frame 3). This current sheet then grows in time as the driving continues forming a thick belt around the closed flux lobe (left frame 4).

Separator reconnection is responsible here for the reopening of the closed flux and by 30 Alfvén times (left frame 7) practically all the closed flux has been disconnected. Notice, that this opening-flux process is different from the previous case where the opening occurred through separatrix-surface reconnection. Furthermore, in this case the separator reconnection occurs in a current sheet that is laid horizontal near its centre as opposed to vertical as it is in the closing separator reconnection situations. This makes it look more like the current sheet associated with separatrix-surface reconnection in the previous scenario and arises due to the different directions the flux is advected across the separator line. The current structures at the sides of the box in frame 7 (left) are associated with reconnection occurring across the periodic boundary and are not considered in our results.

Finally, the fourth scenario, Fig. 5 is really a control experiment in which the overlying field is angled at $\theta=\pi / 2$ such that the two sources are initially unconnected and remain unconnected throughout the entire experiment. As the flux lobes of the sources are driven past each other they do not come into contact and there is no forcing of the fieldlines and so no current structures are developed. What can be seen in the left-hand column is a very slight wiggling of the overlying magnetic field due to waves initiated by the advection of the flux sources.

Comparing the potential experiments with each other it is clear that the closing and the re-opening processes occur quickest in the experiment with overlying field angled at $\theta=0$. This is easily explained since in the potential scenarios reconnection is perfect and instantaneous so it starts as soon as the flux lobes come into contact and ends as soon as they have crossed each other's path. This general dependence of the closed flux lasting for longer the near $\theta$ is to $\pm \pi / 2$ also holds for the dynamical experiments and is explained quantitatively in the next section. Simply comparing the dynamical and potential scenarios qualitatively reveals a number of interesting differences between the two models.

- The dynamical magnetic field lines end up being twisted, whereas the potential ones remain untwisted. The reason for this is because reconnection conserves helicity and so in the dynamical experiments the closed field must be twisted such that this constraint is not violated. In the potential experiments, each frame is independent and so this constraint does not apply.

- Inertial effects are, of course, felt in the dynamical experiments which means that they "lag" behind the potential ones as the field cannot respond instantaneously. Furthermore, in the dynamical scenarios, stresses must first develop between the magnetic fields giving rise to current sheets and increased diffusivity before reconnection can start. This implies that the sources and the flux lobes become severely tangled and warped leading to an even greater lag in time in comparison to the potential scenarios.

- The dynamical experiments result in forces and stresses being setup leading to wave propagation, current sheet formation and the general deformation of the magnetic field. Clearly this means that the quantitative behaviour of the potential and dynamical experiments will be different. In the following sections, we investigate these differences. In particular, we study the time evolution of (i) the amounts of flux connecting the sources (Sect. 3.2); (ii) the reconnection rates of closing and re-opening the field and, hence, the total rate of reconnection (Sect. 3.3); and (iii) the internal structure of the flux within the sources to see exactly how and where the reconnection occurs (Sect. 3.4).

\subsection{Variation of closed flux}

As shown above the time evolution of the percentage of closed flux between the two opposite-polarity sources varies with different (i) orientations of the overlying field and (ii) driving speed. To investigate quantitatively this time-dependence, the percentage of connected flux from each source is determined by tracing fieldlines from a multitude of evenly spaced footpoints all centred in the sources. The flux threading the immediate area surrounding each fieldline is assumed to all connect to the same place as the fieldline. This assumption is reasonable provided the surrounding area is much smaller than the total area of the source. Each fieldline, therefore, has a flux associated with it and can be thought of as a thin flux tube. The flux tubes do not all have the same flux strength, since the internal flux in the sources is not uniform, as already discussed in Sect. 2. In each time step, we trace more than 12500 flux tubes from each source with the maximum flux associated with a tube just 0.03 percent of the total source flux.

For each of the experiments that have closed flux at some point in time Fig. 6 shows plots of the temporal variation of this closed flux as a percentage of the flux in the sources. The temporal variations in closed flux for the dynamical and potential varying overlying field angle experiments are shown in Figs. 6a and $6 \mathrm{~b}$, respectively, whilst the equivalent graphs for the varying driving speed results are shown in Figs. 6c and 6d. There are a number of key features that are immediately evident from these graphs:

- Firstly, the potential curves are all highly symmetric, whereas the dynamical ones are asymmetric with a faster rise phase than fall phase. This implies that in the potential experiments the rate of closing the field is the same as 

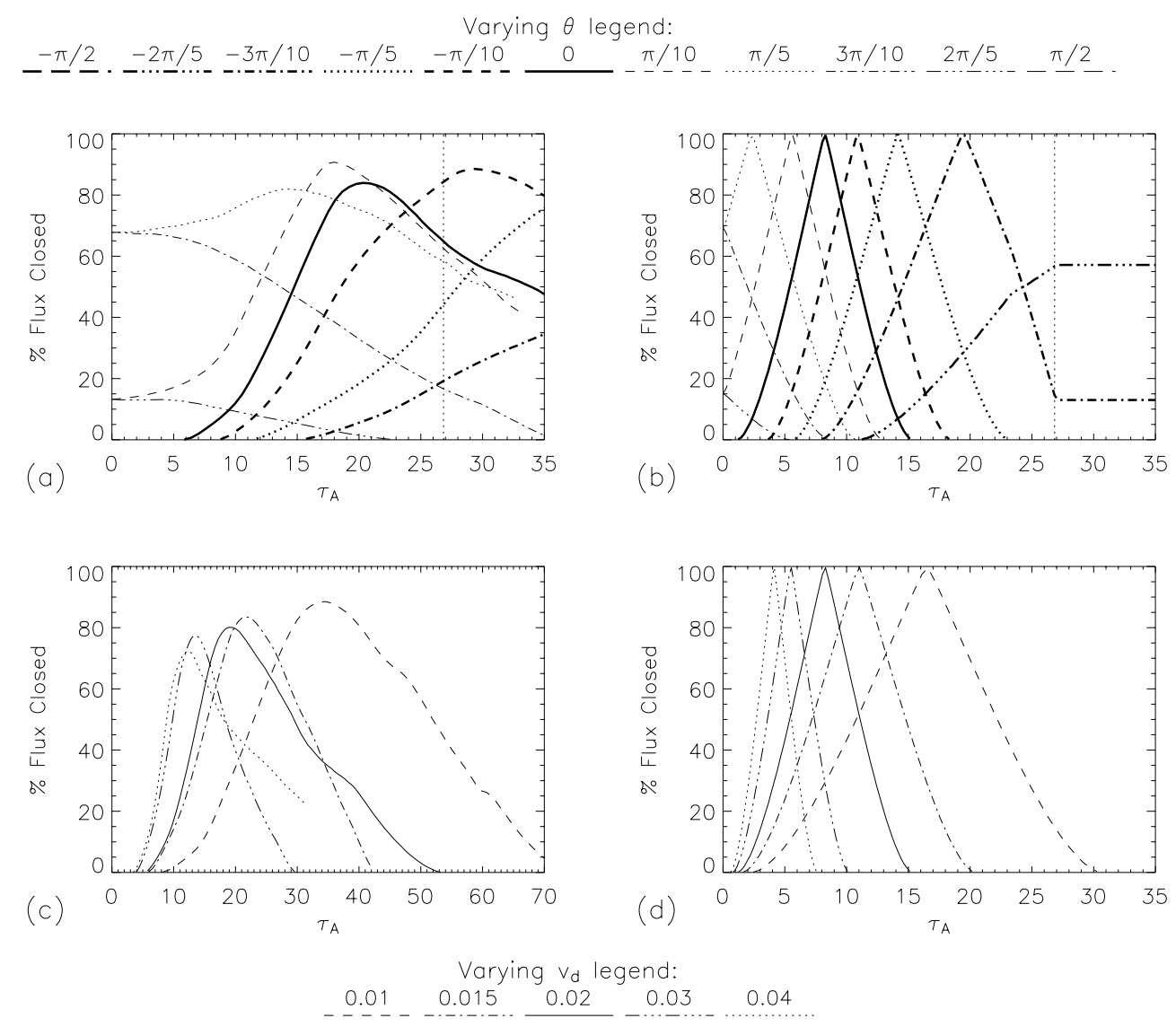

Fig. 6. Closed flux, as a percentage of the total source flux versus Alfvén crossing time for the a) dynamical and b) potential varying overlying field angle experiments (see top legend for $\theta$ values) and c) dynamical and d) potential varying driving speed experiments (see bottom legend for velocities). The vertical line in $\mathbf{a}$ ) and $\mathbf{b}$ ) indicates the time at which the driver is switched off in those experiments.

the rate of re-opening the magnetic field. However, in the dynamical experiments the rate of closing is clearly more rapid than the rate of re-opening. This is related to differences in the reconnection regimes that close or open the magnetic field and is discussed in more detail in the next section.

- Secondly, since reconnection in the dynamical experiments is not instantaneous, as it is in the potential ones, it is not surprising that the time taken to complete the process of closing or re-opening the field is longer in these experiments.

For the potential experiments, the perpendicular crosssection of the flux lobes has roughly the form of a semicircle. Furthermore, since the sources are initially unconnected and the system is in magnetic pressure balance, if we make the simplifying assumption that the magnetic field, in a cross section of the flux lobe some distance from its source, is uniform and with strength equal to the overlying field $B_{\mathrm{h}}$, then the radius of the cross section, $r_{\mathrm{fl}}$, equals

$r_{\mathrm{fl}}=\sqrt{\frac{B_{0} r_{0}^{2}\left(\pi^{2}-4\right)}{\pi^{2} B_{\mathrm{h}}}}$,

where $B_{0}$ is the peak normal component of the magnetic field in the sources and $r_{0}$ is the radius of the sources. Hence, since in the experiments $B_{\mathrm{h}} / B_{0}=0.12$ and $r_{0}=0.065$, it is estimated that $r_{\mathrm{fl}}=0.14$, which, despite the assumptions, is within a few percent of the radius found in the potential experiments. Moreover, since, in the potential experiments, the closed flux lasts for as long as it takes for the two flux lobes to pass through each other and the lobes are at an angle $\theta$ to the normal of the driving direction, the duration of the closed flux period can be estimated and is found to be

$\tau_{\text {pcon }}=2 r_{\mathrm{fl}} / v_{\mathrm{d}} \cos \theta$.

Thus the nearer $\theta$ is to $\pm \pi / 2$ the longer the closed-flux phase lasts.

None of the dynamical experiments complete both the closing and opening processes during the driving period and so it is not possible to say how this relationship varies for these experiments.

- The third thing to notice it that all the dynamical experiments experience a delay in onset of reconnection greater than that in the potential experiments. Using the same simplistic model as above, the onset of reconnection in the potential experiments is estimated to occur at

$\tau_{\mathrm{pst}}=\frac{\left(x_{1}-x_{0}\right) \cos \theta-\left(y_{1}-y_{0}\right) \sin \theta-2 r_{\mathrm{fl}}}{2 v_{\mathrm{d}} \cos \theta}$, 
where the sources are initially situated at $\left(x_{0}, y_{0}\right)$ and $\left(x_{1}, y_{1}\right)$. This comes from the fact that in the potential case the sources are fully connected when the axis through them is aligned and that this will occur at exactly half way through the closed-flux period. Again the potential results follow this curve closely. More interestingly though is that the times of onset in the dynamical experiments also have a similar dependence on $\theta$ and $v_{\mathrm{d}}$. In particular, they appear to satisfy,

$$
\begin{aligned}
\tau_{\mathrm{st}}= & \tau_{\mathrm{pst}} \\
& +\frac{\left(2.13 v_{\mathrm{d}}+0.05\right) \cos \theta+\left(2.30 v_{\mathrm{d}}-0.10\right) \sin \theta}{v_{\mathrm{d}} \cos \theta} .
\end{aligned}
$$

Hence, the onset in the start of the closed-flux period is delayed in the dynamical experiments. This delays occurs because reconnection in MHD only kicks in after currents have built up leading to an enhanced resistivity. The time to build up these currents is, of course, dependent on the rate at which the magnetic field is advected into the system. On top of this the sources in the more slowly driven experiments first start to connect at a slightly greater separation (i.e., relatively earlier) than the more quickly driven sources. This is, of course, associated with the relative speed of flux transport in comparison to the travel time of information in the system and shows that, not surprisingly, if sufficient warning is given reconnection can start relatively earlier.

- Finally, all the potential curves peak at $100 \%$ closed flux, whereas the dynamical ones peak at at most $90 \%$. This has several implications.

- The peak fraction of closed flux scales inversely with driving velocity. The peak fraction of closed flux (for the experiments with same numerical resolution) can be fitted, as a function of the driving velocity, by the curve

$$
Q=\frac{1}{8.6 v_{\mathrm{d}}+1} \text {. }
$$

This shows that complete connectivity is only reached if the driving velocity is exactly zero, whilst for all other cases the maximum closed flux is always less than $100 \%$. This clearly indicates the importance for taking into account dynamical effects when investigating similar types of processes. Potential models can therefore only give us an indication of the changes in the structure of magnetised plasmas as they are stressed. At the other end of the scale, it suggests that if the sources are driven too fast no reconnection occurs at all as the system has no time to react.

- The peak fraction of closed flux also scales with the angle of the overlying field with the peak closed flux decreasing as $\theta \rightarrow \pm \pi / 2$. However, the exact relationship is not clear as we have insufficient results to determine one.

- Clearly, it suggests that the processes of closing and reopening the magnetic field do not overlap in the potential experiments, but may well overlap in the dynamical ones. To investigate if this is indeed true the percentages
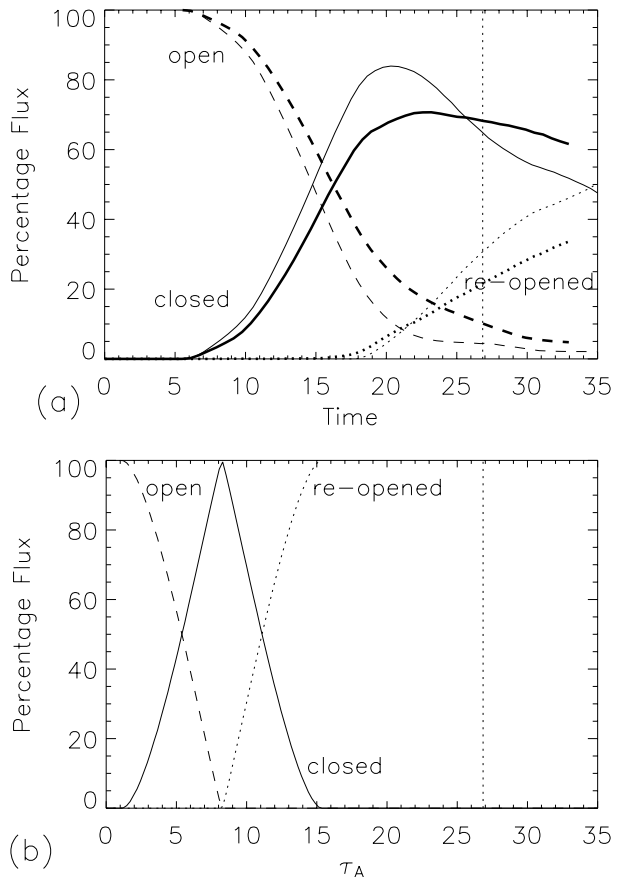

Fig. 7. Percentages of closed (solid), open (dashed) and re-opened (dotted) flux from the positive source versus time for the a) dynamical and b) potential experiment with overlying field angle $\theta=0$. The vertical lines in both plots show the time at which the driver is switched off. In the left-hand panel the thin lines represent the $128 \times 128 \times 65$ numerical resolution run, while the thick lines represent the $256 \times 256 \times 130$ numerical resolution run.

of open, closed and re-opened flux from the positive source are plotted against time for the $\theta=0$ experiment (Fig. 7). In Fig. 7b, which shows the evolution of the potential flux, the percentage of open and re-opened flux are zero when the closed flux is at its maximum, hence the closing and re-opening processes do not overlap. However, for the dynamical case (Fig. 7a) the percentage of open flux is just over $10 \%$ when the closed flux is at its maximum and the amount of re-opened flux is about $5 \%$. At this stage the re-opening process has been going for only 2 Alfvén times, but the closing process does not end for another 10 or more Alfvén times.

This confirmation of overlap of the two reconnection processes in the dynamical experiments implies that one part of a source can be closing whilst another part is simultaneously re-opening the closed field. Thus, the internal evolution of the flux within the sources is quite complex in the dynamical experiments and is studied in detail in Sect. 3.4. Furthermore, this means that simply considering the rate of change of connected flux between sources is not an accurate measure of the total rate of change of flux and, hence, the rate of reconnection. The rates of reconnection are estimated for each experiment in the next section.

As a check, in Fig. 7a, not only are the results from the $128 \times 128 \times 65$ resolution run plotted (thin lines), but the results from an identical experiment with resolution $256 \times 256 \times 130$ are also shown (thick lines). 

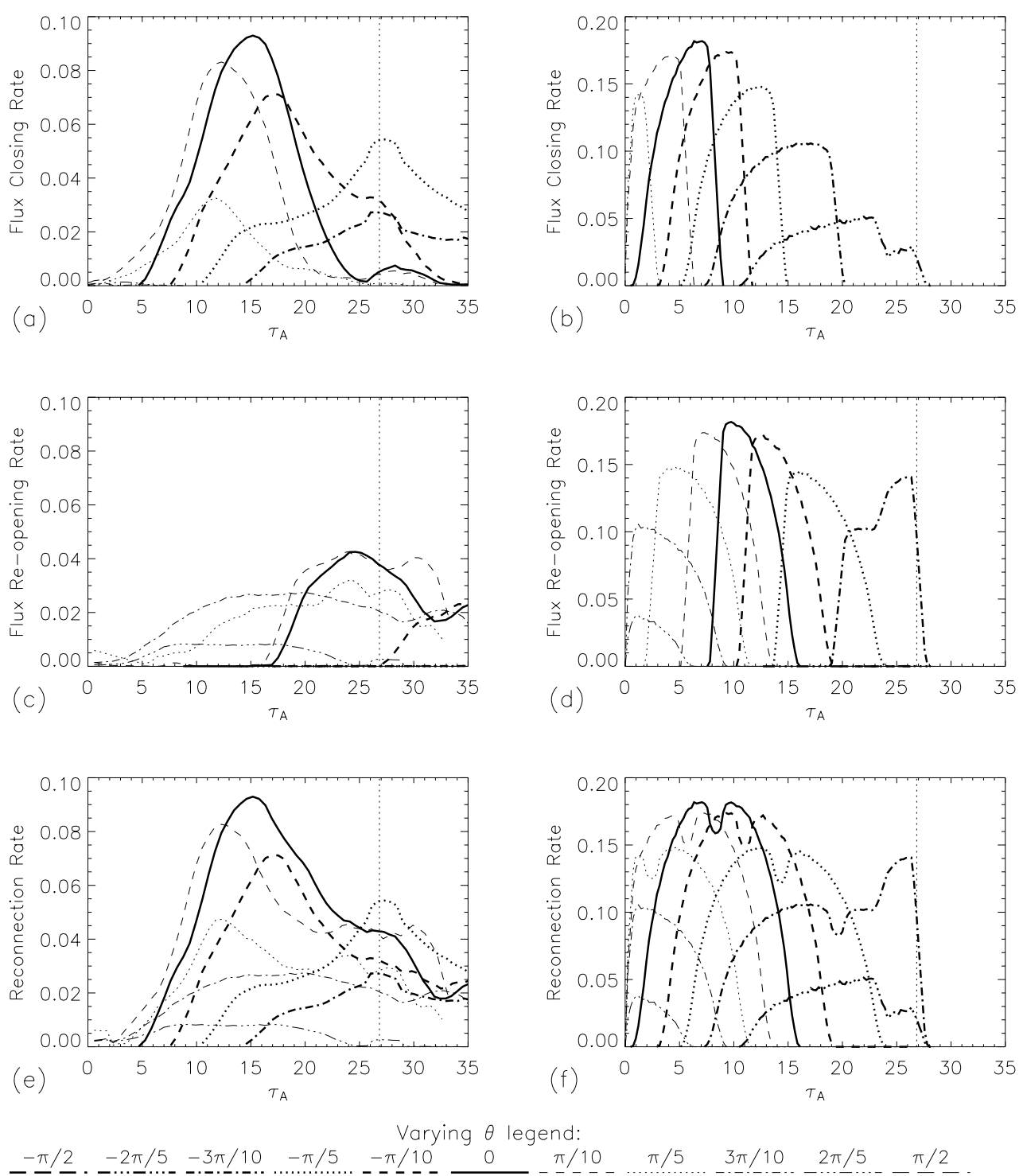

Fig. 8. Temporal variation in the dimensionless rates of reconnection for the varying overlying field angle experiments due to a) and b) closing and c) and d) re-opening the magnetic field and e) and f) the total reconnection rate. The dynamical results are shown in the graphs on the left and the potential results in those on the right. All rates have been normalised with respect to the total flux of the source over one Alfvén time. The vertical line indicates the time at which the driver is switched off.

From this it is clear that there are only minor differences between the two runs and these differences do not significantly affect the general conclusions obtained from the low resolution run. Thus the results in the rest of this paper are therefore based simply on a series of low resolution experiments.

- It is worth noting another feature that appears in many of the graphs, to differing degrees. This is the small dip in the closed flux about $4-6$ time units after the driver has stopped. This is approximately the same time lag as that seen at the start of the experiments before the flux starts closing and is the time scale for information to cross the domain. It can, therefore, be thought of as a global reaction time for the whole system. In particular, in the most rapidly driven case the rate at which the field is opening decreases after this dip.

\subsection{Flux reconnection rates}

To determine the rate of change of flux reconnection, we need to find the amount of flux that is both closing and opening over time. These rates are determined by integrating the curves of the temporal variations in closed and re-opened flux, respectively. They are non-dimensionalised with respect to the total source flux divided by one Alfvén crossing time. The results are shown for the experiments with varying $\theta$ in the 6 graphs of Fig. 8 and for varying $v_{\mathrm{d}}$ in the 6 graphs of Fig. 9. In both of these figures the three graphs on the left refer to the dynamical experiments and the three on the right refer to the potential ones. The graphs in the top rows show the evolution of the flux closing rates, those in the middle rows show the flux opening rates and the bottom rows of graphs shows the total flux reconnection rates (the sum of the closing and re-opening rates). The 

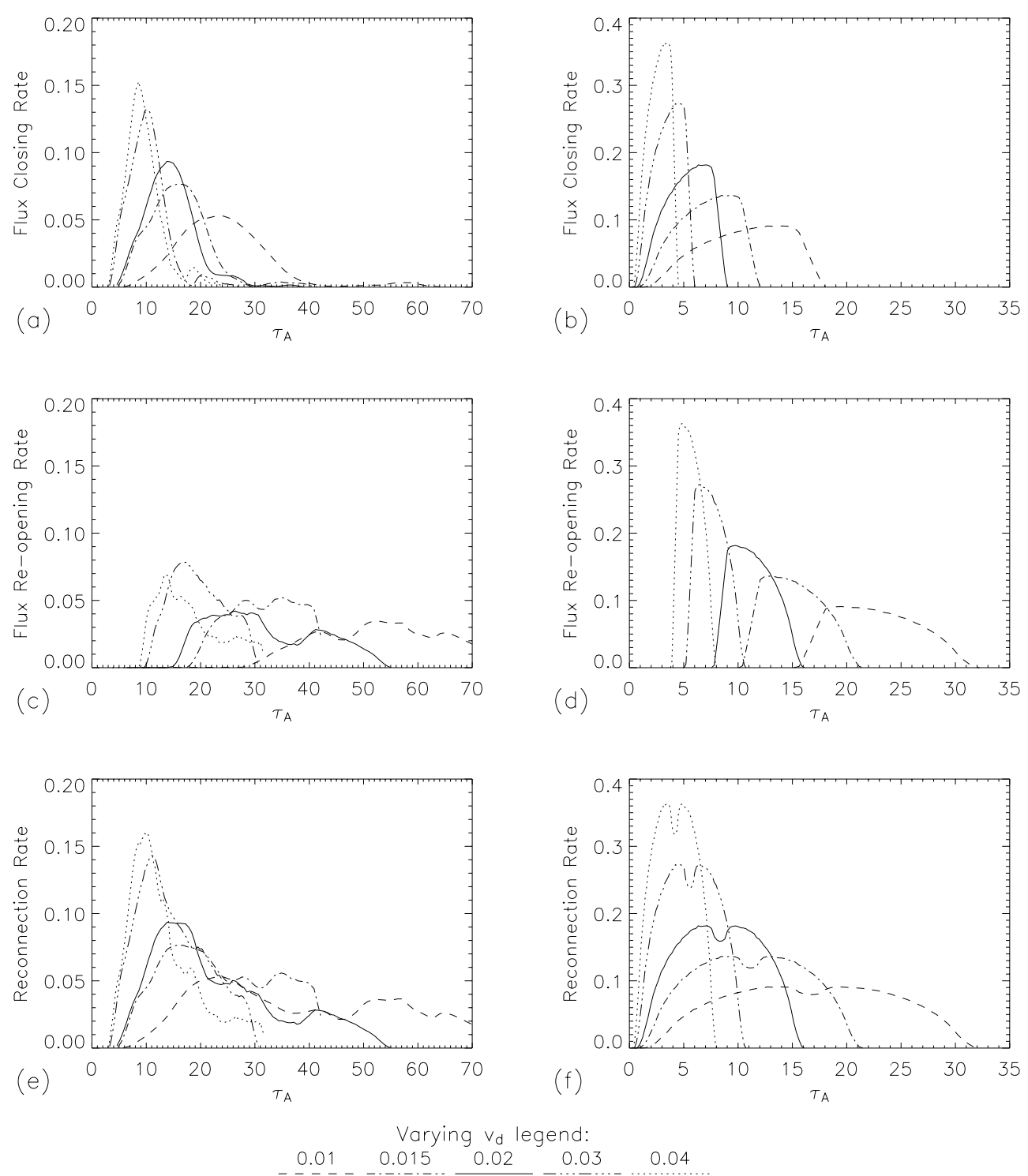

Fig. 9. As Fig. 8 except the graphs show the results for the experiments with varying driving speed.

potential experiments have flux closing and flux opening rates that are effectively mirror images of each other, as one would expect. In the graphs there appears to be a very slight overlap in the two processes; this is due to the use of discrete data points and a finite-difference routine to determine the rates of flux reconnection. In Fig. 8, two of the potential experiments have reconnection occurring at around 23 Alfvén times and show strange behaviour after this time. This is due to the fact that the sources, at this time, have moved near to the edges of the box and are feeling the effects of the closed vertical boundaries in the potential experiments.

In comparison, the dynamical closing and opening flux rates are very different. First, they are definitely not symmetric clearly showing that the process of re-opening the field is not the reverse of closing it. Second, the rate of closing the field through separator reconnection is much more rapid, by roughly a factor of two, than the rate of opening the field through separatrix or separator reconnection. This difference in the dynamical closing and opening rates can be explained by considering the structure of the current sheets involved in these reconnection processes. The closing process involves separator reconnection, where a twisted current sheet is formed along the separator. The flux lobes either side of it are forced into each other such that they become locked together, currents build up and eventually drive reconnection. In such a situation, a tremendous forcing occurs causing strong driving of the reconnection. On the other hand, in the opening process the situation is very different. In the case of the separatrix-surface opening, the current sheet lies on top of the closed flux lobe and the overlying field is principally driven across this lobe rather than being forced into it. The overlying field can simply rise up and slip over the lobe since neither ends of the fieldlines are line tied. Hence, in this situation there is little forcing of the flux systems together and the reconnection is weakly driven. For the separator opening process, the current is generated in a manner similar to the closing, but here the forcing is much weaker as the connected flux can more easily adjust its orientation relative to the source location and the reconnection depends on the inflow of the overlying field. 
The sum of the closing and opening rates is equal to the total rate of reconnection which, in the potential case, is a double peaked curve. If the data had been continuous, rather than finite, a single peak in the middle would probably have occurred, with the peak rates of reconnection occurring for the closed process at the very end, and for the opening process at the very start, of the respective process. The heights of the two peaks are basically the same thus the maximum rates of closing and opening the field in the potential experiments are equal.

The total reconnection curves for the dynamical experiments are very different from the potential ones. They are single peaked and asymmetric showing a steep rise phase and a long fall phase. Obviously, in these curves the peaks, in general, coincide with the peaks in the closing rate since the rate of closing is much more efficient than the rate of opening. In the few cases where this does not hold the process of closing either does not occur at all or is only observed for a short period and therefore does not reach its maximum rate.

An estimate for the total rate of flux reconnection from our simplified analytical potential model can be determined by considering the geometry of the system. The fraction of closed flux from our simple analytical model has the form

$F_{\text {closed }}=\frac{2}{\pi}\left\{\begin{array}{cc}\alpha-\sin \alpha \cos \alpha & \tau_{\mathrm{pst}}<t<\tau_{\mathrm{mx}} \\ \beta-\sin \beta \cos \beta & \tau_{\mathrm{mx}}<t<\tau_{\mathrm{pst}}+\tau_{\mathrm{pcon}}\end{array}\right.$,

where

$\alpha=\arccos \left(1-B\left(t-\tau_{\mathrm{pst}}\right)\right), \quad \beta=\arccos \left(B\left(t-\tau_{\mathrm{mx}}\right)\right)$,

$B=2 / \tau_{\mathrm{pcon}}$ and $\tau_{\mathrm{mx}}=\tau_{\mathrm{pst}}+\tau_{\mathrm{pcon}} / 2$.

Since in the potential evolution the closing and opening phases do not overlap, the rate of reconnection is simply equal to the time derivative of $F_{\text {closed }}$ and thus equals

$$
\begin{aligned}
R= & \frac{4 v_{\mathrm{d}} \cos \theta}{\pi r_{\mathrm{fl}}} \\
& \times\left\{\begin{array}{r}
\left(1-\left(1-v_{\mathrm{d}} \cos \theta\left(t-\tau_{\mathrm{pst}}\right) / r_{\mathrm{fl}}\right)^{2}\right)^{1 / 2} \\
\tau_{\mathrm{pst}}<t<\tau_{\mathrm{mx}} \\
\left(1-\left(v_{\mathrm{d}} \cos \theta\left(t-\tau_{\mathrm{mx}}\right) / r_{\mathrm{fl}}\right)^{2}\right)^{1 / 2} \\
\tau_{\mathrm{mx}}<t<\tau_{\mathrm{pst}}+\tau_{\mathrm{pcon}} .
\end{array}\right.
\end{aligned}
$$

Between $\tau_{\mathrm{pst}}<t<\tau_{\mathrm{mx}} R$ equals the rate of closing and between $\tau_{\mathrm{mx}}<t<\tau_{\mathrm{pst}}+\tau_{\mathrm{pcon}}$ it equals the rate of re-opening. This estimate is reasonably close to the experimentally determined potential reconnection rate despite the assumption that the field in the lobe is uniform which is only true to first order. Furthermore, the estimated analytical peak reconnection rate, although lower than that determined experimentally, still follows the same behaviour as the experimental peak potential reconnection rate. In deed, the analytical peak reconnection rate is

$R_{\mathrm{pmx}}=\frac{4 v_{\mathrm{d}} \cos \theta}{\pi r_{\mathrm{fl}}}=8.79 v_{\mathrm{d}} \cos \theta$,

and is linearly dependent on $v_{\mathrm{d}} \cos \theta$. All the peak total reconnection rates from the potential experiments are plotted in Fig. 10 against $v_{\mathrm{d}} \cos \theta$. Clearly, they all lie on a straight line
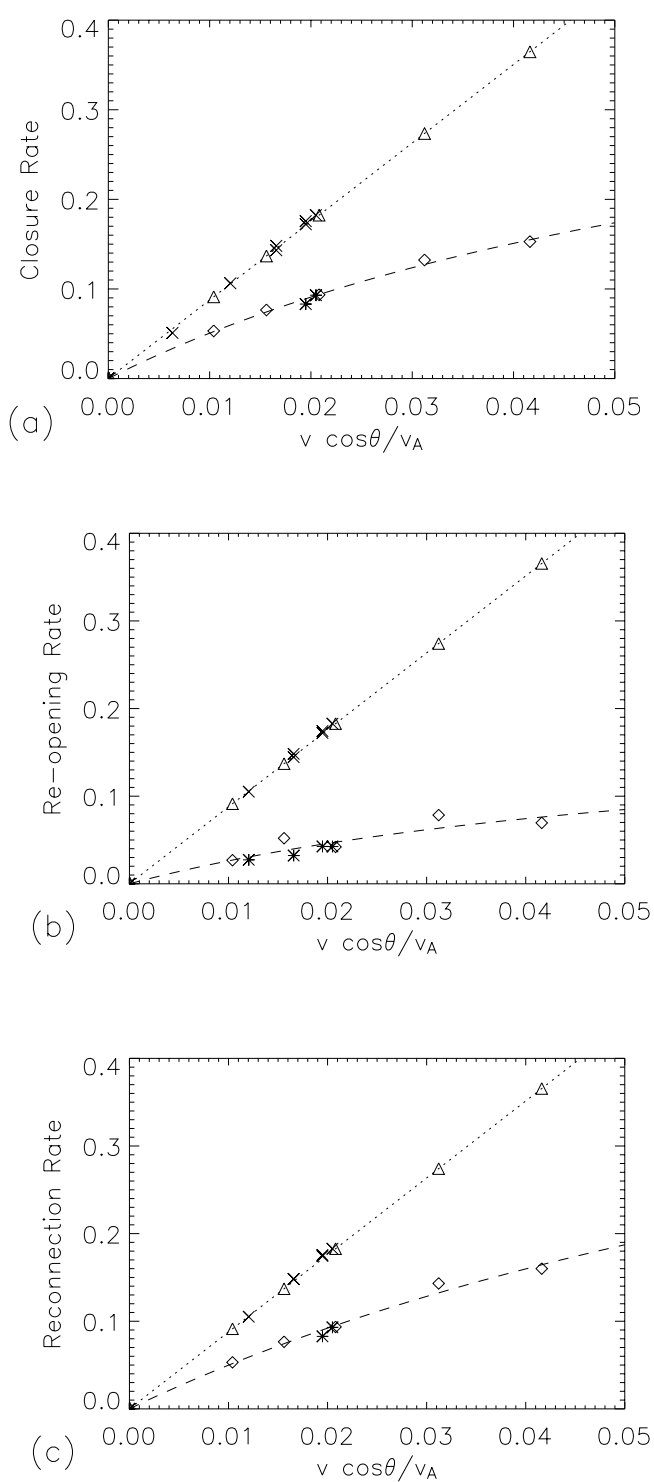

Fig. 10. Peak rates of a) closing, b) re-opening and c) total reconnection against $v_{\mathrm{d}} \cos \theta / v_{\mathrm{A}}$ for the dynamical experiments with varying angle (stars) and varying driving velocity (diamonds) and for the potential experiments with varying angle (crosses) and varying driving velocity (triangles).

which has the form $8.77 v_{\mathrm{d}} \cos \theta$ (dotted line in Fig. 10), which is essentially the analytically predicted line. This is because the rate of reconnection is proportional not to the driving velocity itself, but the component of the driving velocity perpendicular to the current sheet which is aligned with the magnetic field (in particular here it is aligned with the overlying field). Note, though, that the reconnection rates from the experiments are not all as reliable as each other since we only observe (part of) the closing or re-opening process for a short period in some experiments. In these situations, we do not plot these points. If, however, we consider the curves in the dynamical graphs then a different relationship appears to hold. It is clear from the varying velocity peak reconnection rates that they follow asymptotic curves and thus tend towards a maximum reconnection rate. The varying overlying field angle results are not entirely clear, as we have been unable to follow the evolution long 
enough in many of the experiments to get reliable closing/reopening/reconnection rates, but, if we assume that it is the perpendicular driving velocity that is important, then the curves that best fit all these points are

$R_{\mathrm{cmx}}=\frac{0.44 v_{\mathrm{d}} \cos \theta}{v_{\mathrm{d}} \cos \theta+0.08}$,

for the peak closing rates,

$R_{\mathrm{omx}}=\frac{0.19 v_{\mathrm{d}} \cos \theta}{v_{\mathrm{d}} \cos \theta+0.06}$,

for the peak opening rates and

$R_{\mathrm{mx}}=\frac{0.60 v_{\mathrm{d}} \cos \theta}{v_{\mathrm{d}} \cos \theta+0.11}$

for the peak total reconnection rates. These curves are plotted as dashed lines on the respective graphs in Fig. 10. Hence, as $v_{\mathrm{d}} \rightarrow v_{\mathrm{A}}$ the maximum dynamical rate of flux reconnection for closing is 0.45 , for re-opening is 0.20 and for total reconnection is 0.68 . However, more importantly, by comparing these curves with the potential curves, it is clear that the slower the sources are driven the closer the peak dynamical reconnection rate gets to the peak potential rate. In deed, at $v_{\mathrm{d}} / v_{\mathrm{A}}=0.04$ the peak closure rate is $42 \%$ of the potential rate, whereas the peak re-opening rate is just $19 \%$ of the potential rate. However, at $v_{\mathrm{d}} / v_{\mathrm{A}}=0.01$ the dynamical closing and re-opening rates are $58 \%$ and $29 \%$, respectively, of their potential equivalents. This indicates that both the separator and separatrix reconnection rates are fast reconnection processes.

\subsection{Internal source flux evolution}

We have already noted that the source fluxes may simultaneously be closing and re-opening in the dynamical experiments with an overlap of about 8-10 Alfvén times. Thus the internal evolution of the source fluxes is likely to be complex. In this section, we therefore investigate this internal evolution of the fluxes in detail.

To determine the behaviour of the internal flux, we focus on one source and consider thin flux tubes, as in the previous sections, and trace them to find out where they end up. The footpoints of the thin flux tubes are frozen into the plasma and move with the source. Therefore, as the sources are driven past each other, we can monitor which of the thin flux tubes are initially open and stay open, which ones close and which ones re-open. In Fig. 11, the first column of graphs indicate the positions of the sources at the times given at the top of each graph, approximately at intervals of four Alfvén crossing times. The other graphs are arranged in three sets of 9 frames taken at the same times as the graphs in the first column and show the footpoints of the flux tubes traced from inside the solid-ringed source. The footpoints are colour-coded according to where they end up. If the thin flux tube at time, $t=0$, is open and it remains open its footpoint is shaded grey, if the thin flux tube is closed its footpoint turns black and if the thin flux tube has re-opened after being closed its footpoint turns white. The three sets of graphs shown are from the dynamical experiments and have overlying field angles $\theta=-\pi / 5,0$ and $3 \pi / 10$ and $v_{d}=0.02$ and are the same as the three experiments studied in Figs. 2-4.

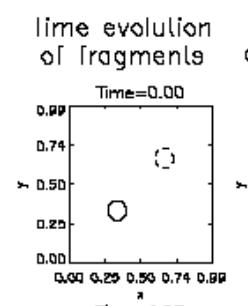

Overlying rield

Overlying rield Overlying rield or Tragments angle: $0 \pi / 5$ angle: 0 angle: $03 \pi / 10$
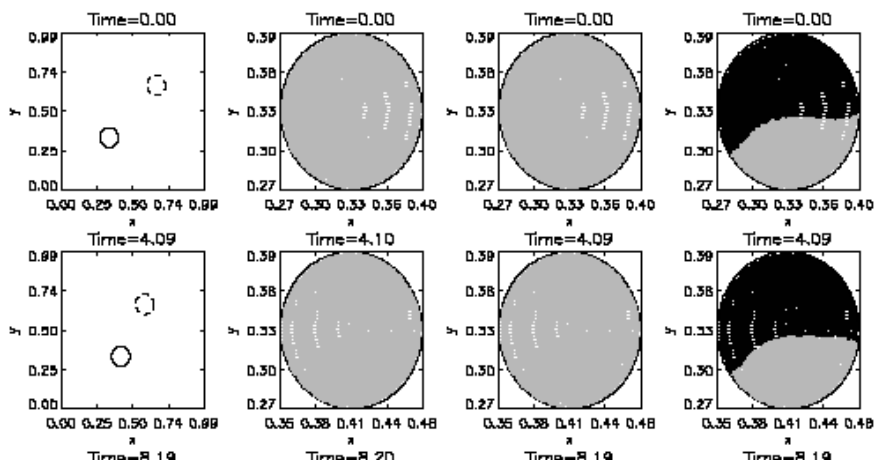

5.270 .530 .330 .360 .40
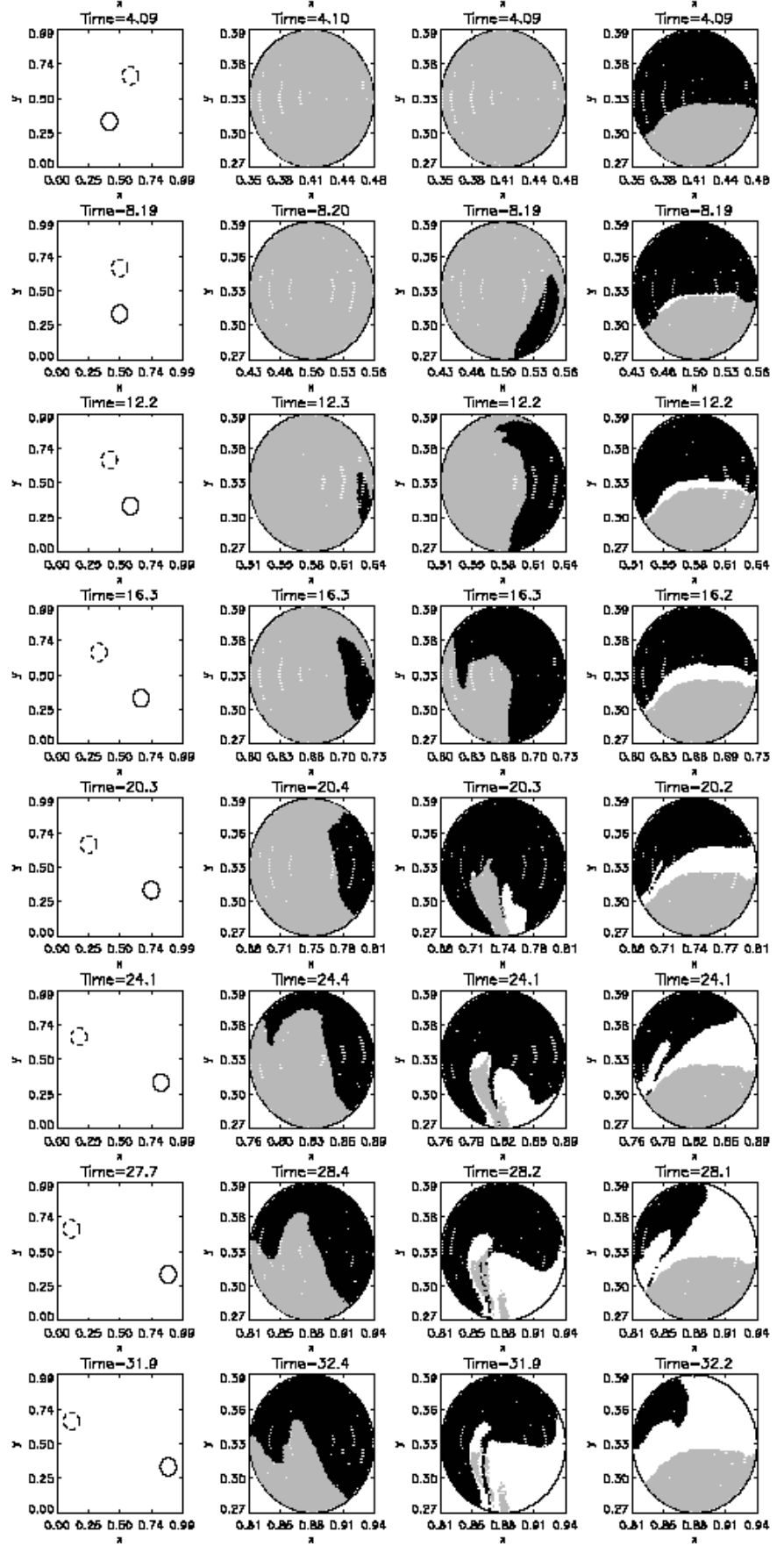

Fig. 11. Time evolution of the internal flux of three dynamical experiments. Column 1: position of the two sources (solid and dashed rings). Columns 2-4: start points of fieldlines from inside the solid-ringed source for experiments with $\theta=-\pi / 5,0$ and $3 \pi / 10$. Start points of flux tubes are colour coded: open field - grey, closed field - black and re-opened field - white.

They show, respectively, situations where the magnetic sources simply close, both close and re-open and just re-open. 


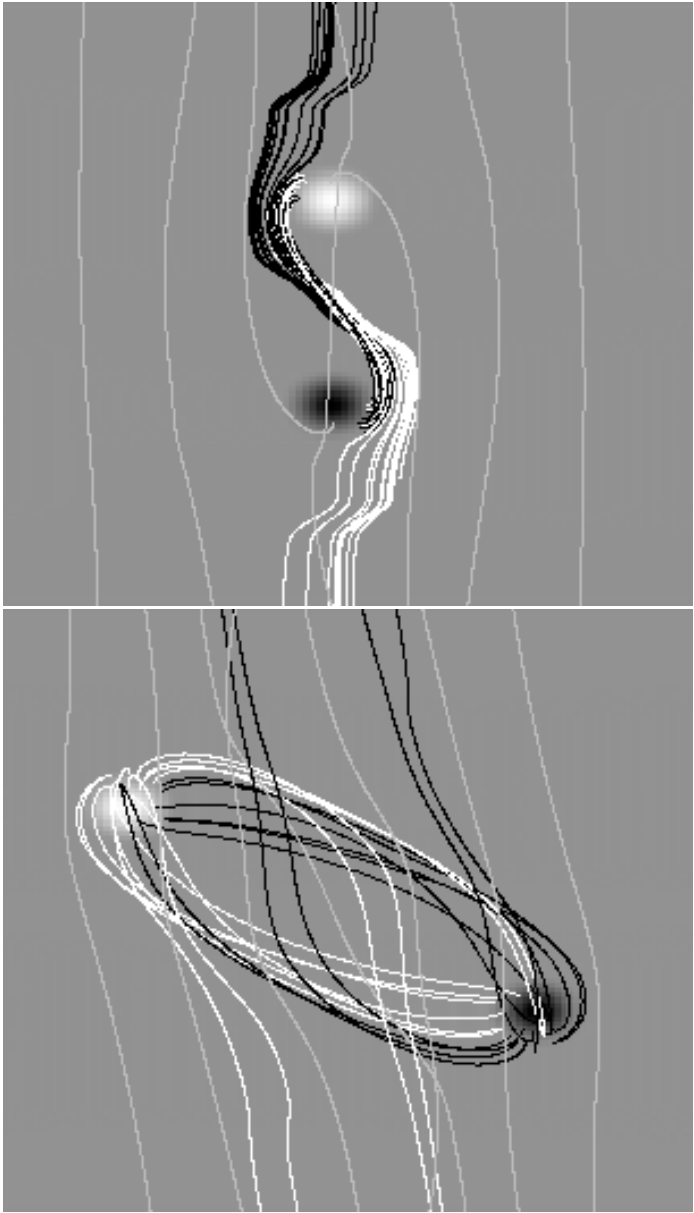

Fig. 12. Magnetic fieldlines originating on the leading edge of each source from the dynamical experiment with overlying field $\theta=0$ showing (top) the initial closing of the magnetic field (at 8 Alfvén times) and (bottom) the initial re-opening of the closed field (at 20 Alfvén times). The white and black fieldlines start from the white and black sources, respectively.

For overlying field angled at $\theta=-\pi / 5$ (Fig. 11, Col. 2) the evolution of the internal flux is fairly straight forward. The flux starts to close when the sources are co-aligned with the overlying field, hence they have been driven past the point at which they are physically closest to each other. In deed, in the potential scenario at this point the sources would be fully connected. The flux on the leading far edge of the source is the first to close. This is the flux whose foot points are almost the furthest from the other source, but it connects because it is the flux that lies on the leading side of the separatrix surface and, hence, is the flux that first comes into contact with the separatrix surface from the other source. Figure 12 (top) is a plot showing white fieldlines traced from the leading edge of the "white" source and the equivalent black fieldlines from the "black" source. The fieldlines in this frame are clearly entwined about each other indicating why the separator current sheet formed is twisted. As the sources are driven further the flux originating from the leading edge of the sources closes such that by the end of the experiment it is only the flux on the trailing side of the source that remains open.
The $\theta=0$ experiment behaves in a similar way (Fig. 11, Col. 3). The flux starts to close when the sources are aligned parallel with the overlying field and the first flux to close is that on the leading far edge of the source. After this the region of closed flux grows in the same way as before until the sources are aligned at about 45 degrees to the overlying field and the driver. At this stage the flux starts to re-open from the far side of the source part way between the leading the trailing sides. This region of open flux grows both inwards, towards the centre of the source, and around the leading side of the source. Its growth is not as smooth as that of the closed region with some fine-scale structure developing within the source. This could result from multiple sites of reconnection within the separatrix current sheets. Figure 12 (bottom) shows a plot indicating the configuration of fieldlines from the far side of the sources at the time that the flux first starts to re-open. The pairs of black and white open fieldlines bent around each other in the middle of the frame are original open fieldlines that have yet to close. The pairs of black and white fieldlines in the top right and bottom left of the frame, respectively, are re-opened fieldlines. The reconnection sites for these re-opened fieldlines are likely to be at either end of the separatrix surface surrounding the closed flux. It is not easy to say exactly where the reconnection occurred, but comparing this frame with frame 6 on the left-hand side of Fig. 3 in which two separatrix current sheets are visible is highly suggestive of two reconnection sites.

Finally, for $\theta=3 \pi / 10$ (Fig. 11, Col. 4) the sources start off already partially connected such that the flux just re-opens. Note, however, that the initially connected flux is that on the near side of the source and does not extend far up the leading edge of the source. Hence, when the flux starts to re-open it does so from the middle of the source, not the edge. This region of re-opened flux extends from the middle to either edge of the source with the majority of the re-opening occurring on the leading edge of the source. Again the re-opening is not smooth and fine-scale structure develops for the same reasons as above.

The evolution of the internal flux within the equivalent potential experiments is shown in Fig. 13. The potential evolution is similar to the dynamical evolution in that the initial regions of closed flux all start on the leading edge of the sources and sweep around the nearside with the far trailing side of the source being the last to close. The original site of re-opened flux is also similar being on the leading side of the source in the first two experiments and in the middle on the last experiment. Again these regions grow in a similar way to those in the dynamical experiments, except there is no evidence of fine-scale structure. Thus, the evolution is, of course, much smoother in these potential experiments, but is also much faster too as has already been discussed.

\section{Conclusion}

It is very likely that at least some percentage of the coronal heating budget comes from the release of magnetic energy through driven magnetic reconnection. Typically, we think of magnetic reconnection as being driven by the emergence and divergence of new flux (such as in X-ray jets and X-ray bright points above ephemeral regions) or by the convergence and 


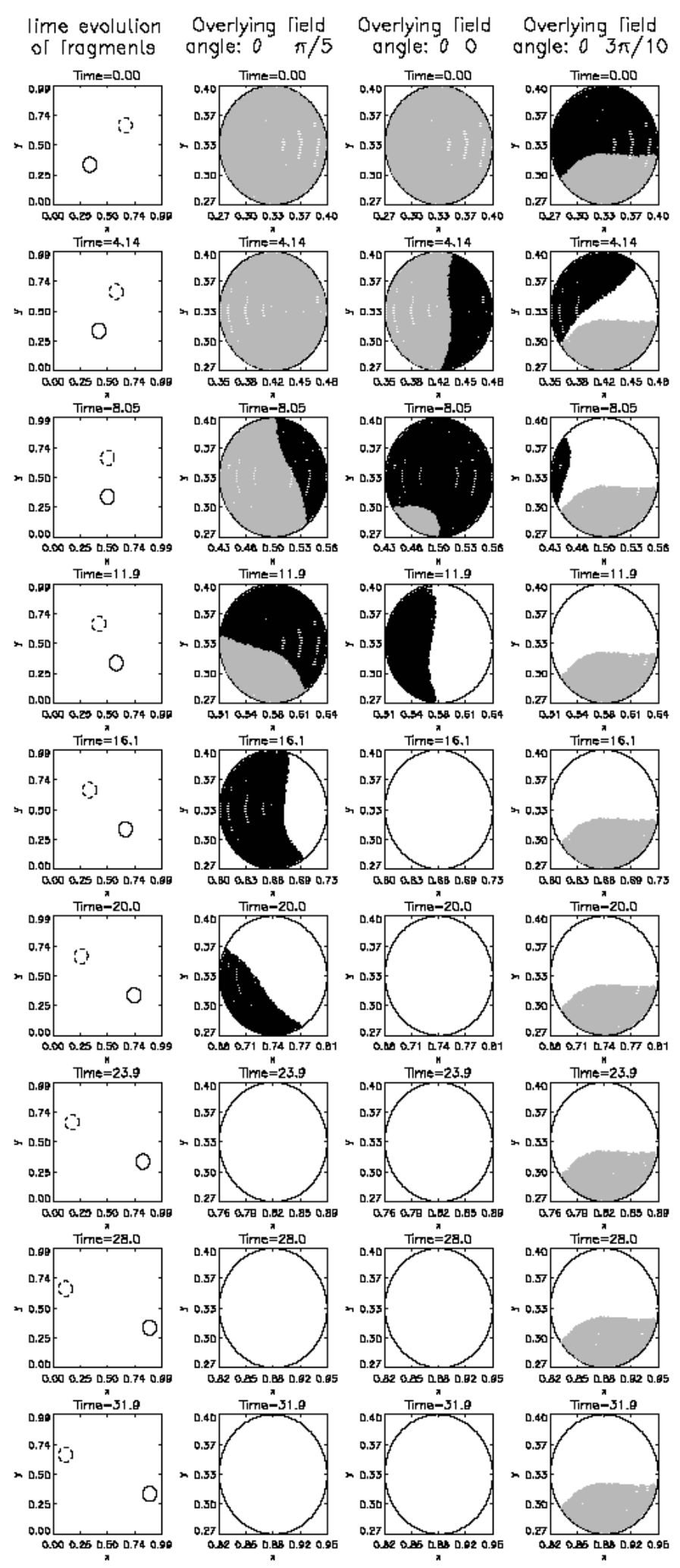

Fig. 13. As Fig. 11 except here the time evolution of the internal flux of the equivalent three potential experiments is shown.

cancellation of unconnected magnetic sources (such as X-ray bright points associated with cancelling magnetic features). In both cases it is the movement phases (i.e., the diverging or converging) of the sources that drives the reconnection rather than the flux creation or destruction phases themselves. For example, it is known that bright points do not appear above ephemeral regions as soon as any new flux is created, but their appearance is delayed by an hour or so. Similarly, X-ray bright points are known to end before all the flux in a cancelling magnetic feature is cancelled (Priest et al. 1994). Thus, reconnection can occur in any situation where unconnected magnetic sources are driven past each other in such a way that at some stage they connect and then, if driven far enough, disconnect again. In such situations, the amount of flux connected is dependent on a number of things including the relative directions of the overlying field and transport of the sources and the speed of transport. These effects have been investigated here with particular attention paid to the rate of reconnection and the evolution of the flux connectivity.

A dynamical model, which solves the non-ideal MHD equations in a three-dimensional domain, is used to investigate the connectivity of magnetic sources that are driven past each other in an overlying field. The following observations are found for these particular experiments:

- The driving of the sources (i) creates a twisted current sheet along the separator between the sources creating closed fieldlines via separator reconnection and (ii) causes separatrix reconnection in an untwisted separatrix current sheet that re-opens the field again. The closed field created is twisted, unlike in the potential experiments, due to the restriction of having to conserving helicity.

- The rates of reconnection are fast. In particular, the rates of closing and re-opening occur at $42 \%$ and $19 \%$ of the instantaneous potential reconnection rate, respectively, if driven at $v_{\mathrm{d}} / v_{\mathrm{A}}=0.04$ and they occur even more efficiently at $58 \%$ and $29 \%$, respectively, if $v_{\mathrm{d}} / v_{\mathrm{A}}=0.01$. Thus, here, the driven separator reconnection rate (rate of closing) is roughly twice as fast as the driven separatrix reconnection rate (rate of opening). This is because during the separator reconnection the two flux system have to pass through each other, i.e., reconnect, as there is no other way around. Whereas in the separatrix-surface reconnection the forcing of the two flux systems is not so great as the two flux systems can slip over each other.

The reconnection in three-dimensions is occurring much faster than one would expect from the predictions of twodimensional reconnection models. The reason for this is because in three-dimensions the current sheet is a surface, not a line, and within this surface there are multiple centres of reconnection allowing many field lines to reconnection at any one instance.

- The rates of reconnection are all dependent on the direction of driving perpendicular to the current sheet which in these experiments is aligned with the overlying field. In the experiments, the peak dimensionless reconnection rates were found to follow asymptotic curves and thus they tended to a maximum dimensionless rate, as $v_{\mathrm{d}} \rightarrow v_{\mathrm{A}}$. Maximum rates of $0.45,0.20$ and 0.68 (where 1 is equal to the total flux in the sources reconnecting in one Alfvén time) are found 
for the closing, opening and total reconnection processes, respectively.

- Dynamical processes take longer to occur than potential ones, because, in MHD, the processes do not occur instantaneously. Instead, currents must first build up leading to enhanced resistivity before the reconnection can start. Furthermore, once it has started, its rate is highly dependent on the inflow of magnetic flux.

- The processes of closing and opening the magnetic field overlap with the peak closed flux from the sources at anyone time being less than $100 \%$ and (i) varying inversely with $v_{\mathrm{d}}$ and (ii) decreasing as $\theta \rightarrow \pm \pi / 2$. This is due to the geometry of the system which effects the efficiency of the reconnection processes.

- Typically it is found that the sources start to connect when the line through the source centres is coaligned with the overlying field. Hence, even though the two sources are moving further apart they may be creating new closed flux. In contrast, the sources in the potential experiments start to connect earlier than this when their flux lobes first come into contact. The lag in onset of reconnection in the MHD experiments is due to the fact that current sheets (current enhancements) must first develop to enable the resistivity to increase sufficiently for diffusion to become important and hence reconnection to start.

- The dynamical experiments do not relax back to a state equivalent to the initial one from which they start because (i) in MHD, the reconnection processes are not completely efficient and, hence, the opening of the field is not the reverse of closing it and (ii) helicity, injected by the driving of the fragments, is not released during the reconnection processes, thus the magnetic field has a different helicity at the end than when it started.

- The internal flux evolution of the sources shows two important features. (i) The first flux to reconnect is not in the regions of the sources closest to each other, but in the regions whose fieldlines form the leading sides of the separatrix surfaces which are forced into each other, and hence are nearest to where the current sheet is formed. These regions are the same in the closing and re-opening processes. The regions of the sources closest to each other are the regions which remain closed the longest. (ii) There is evidence of fine-scale structure, in particular, during the opening separatrix-reconnection process. This probably results from the fact that there are multiple centres of reconnection. For separatrix reconnection there is no evidence of nulls occurring on the separatrix surface and so the reconnection is more likely to be component wise reconnection.

From these results there are three important conclusions about three-dimensional reconnection for plasma physics in general:

- The rate of reconnection is highly dependent on the rate of driving and the geometry of the system.

- Separator and separatrix reconnection can be fast.

- Driven three-dimensional reconnection rates can tend to a maximum.

Thus three-dimensional reconnection of small-scale magnetic sources is a relatively fast and efficient process and is highly likely to play a role in the heating of the quiet-Sun corona. In particular, interactions similar to the ones analysed in this paper are likely to be occurring throughout the corona due to the continuous movement of the mixed polarity magnetic sources in the quiet Sun and active regions. The sources are continuously being dragged along by the overshoots of convection cells forcing them to first move to the edges of the supergranule cells and then to move along the intergranular lanes. This movement within the complex magnetic environment of the solar atmosphere will mean that the magnetic connections between these sources are frequently changing. However, other than X-ray bright points and nanoflares we see little evidence of these fieldline changes occurring in EUV and X-ray images. This is likely to be because energy is either being released continuously throughout the corona or it is not resulting in plasma of the required observational temperature for the particular instrument used. The flux that is being reconnected is probably slightly less than that that is reconnected in a cancelling magnetic feature or an ephemeral region. Thus, the energy that is released will be lower and possibly spread over a larger area. Hence, the energy from these events could lead to simply maintaining the background coronal temperature of about a million degrees, as the real coronal heating mechanism must do.

Acknowledgements. K.G. would like to thank PPARC for financial support through an advanced fellowship and Carlsbergfondet in the form of a Scholarship over various periods of the project. C.E.P. would like to thank the RAS for supporting her whilst she was the Sir Norman Lockyer Fellow (1998-2001) and PPARC for support on the St Andrews Solar Theory rolling grant and as a PPARC Advanced Fellow. The computational analysis for this paper was carried out on the PPARC funded Compaq MHD Cluster and the joint SRIF and PPARC funded linux cluster, copson, in St. Andrews.

\section{References}

Birk, G., Dreher, J., \& Neukirch, T. 1996, The Solar Atmosphere, ed. R. D. Bentley, \& J. T. Mariska, ASP Conf. Ser., 111, 89

Dreher, J., Birk, G., \& Neukirch, T. 1997, A\&A, 323, 593

Forbes, T. G., \& Priest, E. R. 1984, Sol. Phys., 94, 315

Galsgaard, K., Titov, V. S., \& Neukirch, T. 2003, ApJ, 595, 506

Galsgaard, K., \& Nordlund, Å. 1996, J. Geophys. Res., 101, 13445

Galsgaard, K., \& Nordlund, Å. 1997, J. Geophys. Res., 102, 231

Galsgaard, K., \& Parnell, C. E. 2004, A\&A, in preparation

Galsgaard, K., Parnell, C. E., \& Blaizot, J. 2000a, A\&A, 362, 395

Galsgaard, K., Priest, E. R., \& Nordlund, Å. 2000b, Sol. Phys., 193, 1

Greene, J. M. 1988, J. Geophys. Res., 93, 8583

Hendrix, D. L., \& Van Hoven, G. 1996, ApJ, 467, 887

Lau, Y. T., \& Finn, J. M. 1990, ApJ, 350, 672

Longbottom, A. W., Rickard, G. J., Craig, L. J. D., \& Sneyd, A. D. 1998, ApJ, 500, 471

Longcope, D. W. 1998, ApJ, 507, 443L

Nandy, D., Hahn, M., Canfield, R. C., \& Longcope, D. W. 2003, ApJ, in press

Ng, C. S., \& Bhattachrjee, A. 1998, Phys. Plasma, 5, 11, 4028

Nordlund, Å., \& Galsgaard, K. 1997, Tech. Rep., Astronomical Observatory, Copenhagen University

Otto, A. 1995, J. Geophys. Res., 100-A7, 11863

Parker, E. N. 1987, ApJ, 318, 876 
Parnell, C. E., Priest, E. R., \& Titov, V. S. 1994, Sol. Phys., 153, 217

Priest, E. R., \& Démoulin, P. 1995, J. Geophys. Res., 100-A12, 2344

Priest, E. R., Parnell, C. E., \& Martin, S. F. 1994, ApJ, 427, 459

Priest, E. R., \& Titov, V. S. 1996, Phil. Trans. R. Soc. Lond. A., 354, 2951

Shi, Y., Wu, C. C., \& Lee, L. C. 1991, J. Geophys. Res., 96-A10, 17627

Shibata, K., Nozawa, S., \& Matsumoto, R. 1992, PASJ, 44, 265

Forbes, T. G., \& Priest, E. R. 1984, Sol. Phys., 94, 315

Galsgaard, K., Titov, V. S., \& Neukirch, T. 2003, ApJ, 595, 506

Galsgaard, K., \& Nordlund, Å. 1996, J. Geophys. Res., 101, 13445
Galsgaard, K., \& Nordlund, Å. 1997, J. Geophys. Res., 102, 231

Galsgaard, K., \& Parnell, C. E. 2004, A\&A, in preparation

Galsgaard, K., Parnell, C. E., \& Blaizot, J. 2000a, A\&A, 362, 395

Galsgaard, K., Priest, E. R., \& Nordlund, Å. 2000b, Sol. Phys., 193, 1

Greene, J. M. 1988, J. Geophys. Res., 93, 8583

Hendrix, D. L., \& Van Hoven, G. 1996, ApJ, 467, 887

Titov, V. S., Galsgaard, K., \& Neukirch, T. 2002, ApJ, 582, 1172

Uchida, Y., \& Sakuri, T. 1977, Sol. Phys., 51, 413

Yokoyama, T., \& Shibata, K. 2001, ApJ, 549, 1160

Zhang, M., \& Low, B. C. 2002, ApJ, 576, 1005 